\title{
Assessment of the effectiveness of multiple-stripe analysis by using a stochastic earthquake input model
}

\author{
Fabrizio Scozzese ${ }^{1}$, Enrico Tubaldi ${ }^{2}$, Andrea Dall'Asta ${ }^{1}$
}

\begin{abstract}
${ }^{1}$ School of Architecture and Design, University of Camerino, Viale della Rimembranza, 63100 Ascoli Piceno (AP), Italy; E-mail: fabrizio.scozzese@unicam.it, andrea.dallasta@unicam.it
\end{abstract}

2 Department of Civil and Environmental Engineering, University of Strathclyde, 75 Montrose Street, G1 1XQ Glasgow, UK; E-mail: enrico.tubaldi@strath.ac.uk

\begin{abstract}
Current practical approaches for probabilistic seismic performance assessment of structures rely on the concept of intensity measure (IM), which is used to decompose the problem into hazard analysis and conditional seismic demand analysis. These approaches are potentially more efficient than traditional Monte-Carlo based ones, but the performance estimates can be negatively influenced by inadequate setup choices. These include, among the others, the number of seismic intensity levels to consider, the number of structural analyses to be performed at each intensity level, and the lognormality assumption for the conditional demand. This paper investigates the accuracy and effectiveness of a widespread IM-based method for seismic performance assessment, multi-stripe analysis (MSA), through an extensive parametric study carried out on a three-story steel moment-resisting frame, by considering different setup choices and various engineering demand parameters. A stochastic ground motion model is employed to describe the seismic hazard and the spectral acceleration is used as intensity measure. The results of the convolution between the seismic hazard and the conditional probability of exceedance obtained via MSA are compared with the estimates obtained via Subset Simulation, providing a reference solution. The comparison gives useful insights on the influence of the main parameters controlling the accuracy and precision of the $I M$-based method. It is shown that with the proper settings, MSA can provide risk estimates as accurate as those obtained via Subset Simulation, at a fraction of the computational cost.
\end{abstract}

Keywords: Seismic risk; Reliability; Multiple-stripe analysis; Subset Simulation; IM-based approach; Stochastic model.

\section{Introduction}

Seismic risk analysis aims to assess the probability of a structural system attaining an unsatisfactory performance at least once within a reference time frame. Probabilistic approaches for seismic risk assessment can be grouped in two classes (Bazzurro et al. 1998; Franchin et al. 2012; Bradley et al. 2015): 1) direct, simulation-based approaches; 2) conditional, IM-based approaches (where $I M$ stands for Intensity Measure).

The first class consists of methods based on the observation of the system response to samples drawn from the probability distribution of the random inputs (e.g. earthquake characteristics, structural model). These include Monte Carlo simulation (Rubinstein and Kroese 2017) and the more efficient variance reduction techniques, such as Importance Sampling (Jayaram and Baker 2010) and Subset Simulation (Au and Beck 2003). These methods require a ground motion model from which earthquake samples are generated. Although their usage is limited mainly to the research field, they generally represent the most robust mean for estimating the seismic risk of any complex, even strongly nonlinear system. Their main limit is the high number of numerical analyses needed.

The methods belonging to the second class have been developed in the last 20 years since the seminal works of (Cornell et al. 2002). The main purpose of these methods is to make seismic risk estimation a more practice-oriented and computationally affordable task. A conference paper by (Cornell 2005) clarifies the rationale behind the $I M$-based approaches, which rely on the definition of a specific parameter, named Intensity Measure $(I M)$, describing the ground motion intensity at the site of the structure. By introducing the $I M$, the estimation of the seismic demand hazard, expressing the mean annual frequency (MAF) of exceeding different values of the Engineering Demand Parameter (EDP) of interest, is split into two separate probabilistic steps. The first one is the seismic hazard assessment, which often uses empirical ground motion prediction equations (GMPEs) to provide a statistical description of the $I M$ (e.g., (Bozorgnia et al. 2014)). The second one consists in the evaluation of the seismic demand conditional to specific values assumed by the $I M$. A set of recorded ground motions, accounting for the seismic recordto-record variability, is considered as input for performing the structural analyses at different $I M$ levels. Different methods can be used (Mackie and Stojadinović 2005; Jalayer and Cornell 2009) to carry out this task, the most diffused ones being incremental 1 
dynamic analysis (IDA) (Vamvatsikos and Cornell 2002), cloud analysis (Mackie and Stojadinović 2005; Tubaldi et al. 2016), and multiple-stripe analysis (MSA) (Mackie and Stojadinović 2005; Bradley 2013a). The results of the two steps of the analysis are convolved together to obtain the unconditional demand hazard curves, which is the same result that could be obtained by applying a direct simulation-based approach. The main differences between the two approaches are summarized in Table 1.

Table 1 Main features of $I M$-based and simulation-based probabilistic approaches.

\begin{tabular}{|c|c|}
\hline Simulation-based & IM-based \\
\hline $\begin{array}{l}\text { - } \text { research-oriented } \\
\text { no need of any intensity measure (no } \\
\text { conditioning) } \\
\text { - large number of simulations (structural } \\
\text { analyses) required } \\
\text { - robust and confident tool for seismic risk } \\
\text { estimation } \\
\text { requires a stochastic model for describing } \\
\text { the seismic input. }\end{array}$ & $\begin{array}{l}\text { - } \text { practice-oriented } \\
\text { - need the choice of an intensity measure for } \\
\text { conditioning purposes } \\
\text { - potentially requires a reduced number of } \\
\text { structural analyses if } I M \text { is efficient } \\
\text { - potentially biased if } I M \text { is not sufficient } \\
\text { and ground motion records are not } \\
\text { representative of the hazard } \\
\text { - can be applied using recorded ground } \\
\text { motions, but in this case the accuracy } \\
\text { cannot be checked }\end{array}$ \\
\hline
\end{tabular}

It is noteworthy that the discussion on conditional versus non-conditional methods dates back to 20 years ago (Bazzurro et al. 1998). While many research efforts have been devoted to the development of conditional-based approaches (e.g., (Bradley 2013a; Gehl et al. 2015)), very few studies have focused on their accuracy and have carried out comparisons of the seismic demand and risk estimates obtained with the direct simulation-based ones. Among these, (Bradley et al. 2015) tested different methods for evaluating, through a conditional approach, the peak displacement response of a nonlinear single-degree of freedom (SDOF) system. The results obtained using a plain Monte Carlo simulation-based direct approach were used as reference solution. Franchin et al (Franchin et al. 2012) used the Importance Sampling method to validate some $I M$-based approaches (i.e., IDA and cloud analysis), but this study again considered only a single EDP (i.e., the maximum drift angle of a reinforced-concrete frame) and focused on quite high MAFs of limit state exceedance, up to $10^{-3} 1$ year. These validation studies require resorting to a stochastic seismic input model rather than using GMPEs for the hazard analysis together with real ground motion records for the structural response analysis.

This paper aims to provide an in-depth evaluation of the efficiency and accuracy of the MSA-based conditional approach combined with the widely employed spectral acceleration at the system fundamental period $\left(S_{a}(T)\right.$ ) as $I M$ (Shome et al. 1998; Jalayer and Cornell 2009), assuming that a stochastic ground motion model can provide an accurate representation of the site seismicity. In particular, the objective of the study is to assess the influence of the main setup choices and values of the parameters controlling the method, such as: the number of $I M$-levels ( $I M$-stripes); the number of ground motion samples per stripe; the technique adopted for the computation of the conditional demand model; the size of the whole $I M$ range investigated, hence the truncation of the $I M$ hazard curve.

It is worth noting that although several metrics for $I M$ are available, in this paper $S_{a}(T)$ is employed for two main reasons: 1) $S_{a}(T)$ is widely used not only by researchers but also by practitioners (Porter 2016)(Shome et al. 1998; Jalayer and Cornell 2009); 2) the outcomes of the present work provides useful insights on the expected level of accuracy and precision with conditional approaches, even if the $I M$ chosen is not the most appropriate for the specific case analysed. Details on the sufficiency and efficiency of other, more advanced IMs can be found in the relevant scientific literature (see e.g. (Dávalos and Miranda 2019a)(Kazantzi and Vamvatsikos 2015)(Eads et al. 2015)). The use of these IMs will be also object of future specific investigations.

For the purpose of the present study, a three-storey moment-resisting frame, often considered for investigating the efficiency of seismic response control devices (Gupta and Krawinkler 1999; Barroso and Winterstein 2002; Ohtori et al. 2004; Dall'Asta et al. 2016; Scozzese et al. 2019), is analysed, and seismic demand hazard curves are developed for various EDPs, namely the interstory drifts, absolute accelerations, residual drifts, base shears, and relative displacements. First, a nonlinear SDOF model of the frame is considered, allowing to perform a significant number of analyses in short time and assess the accuracy and precision obtained by varying the controlling parameters in a sufficiently wide range. After this parametric investigation, a multidegree-of-freedom (MDOF) model of the frame is analysed, to evaluate whether the findings also hold for a case in which higher order modes may affect some EDPs of interest. 
Subset Simulation (Au and Beck 2003) is used in the direct approach to obtain a set of reference reliable solutions and thus quantify the estimation errors obtained using the MSA-based conditional approach. The comparison between the two approaches allows investigating the influence of the parameters and the various choices controlling the application of the conditional approach and to provide useful information on their optimal setup. A single source model is used to describe the seismic scenario, and the Atkinson-Silva ground motion model (Atkinson and Silva 2000) is used to generate synthetic earthquake samples at the site of interest. The use of a stochastic ground motion model overcomes the issue of the lack of real ground motions consistent with the site seismic hazard, in particular at high $I M$ levels. In order to obtain information about the statistical precision of the estimates, multiple independent simulations are performed for each system and EDP analysed. The numerical solutions are summarized by considering the average demand hazard curves and the coefficients of variation of the results. This permits to evaluate the potential bias (hence the expected accuracy) and the precision of the solution, compared to the reference one.

Although limited to a single case study, the outcomes of the present investigation provide, along with (Franchin et al. 2012; Bradley et al. 2015), useful insights into the convergence and accuracy properties of conditional approaches, thus helping to exploit in an optimal way their potentialities.

\section{Methodology}

This section briefly describes the probabilistic tools examined in this paper, namely the unconditional approach (Subset Simulation), used to provide the reference solutions, and the conditional approach (MSA). The starting point for both the approaches is the definition of the seismic scenario, which requires a characterization of the potential seismic sources in terms of the probability distribution of the moment magnitude $M$ and epicentral distance $R$. In this work, a single source is considered, and a stochastic ground motion model (Atkinson and Silva 2000) is employed to simulate the propagation of the waves from the source to the site, as detailed in Subsection 3.1. The output of both the unconditional and conditional approaches is $v_{D}(d)$, i.e., the MAF of exceedance of different values $d$ of the demand parameter $D$ (random variable), also denoted as EDP in the literature.

\subsection{Reference solution via unconditional approach}

The evaluation of the demand hazard according to the unconditional approach can be formalized as follows:

$v_{D}(d)=\bar{v} G_{D}(d)$

where $\bar{v}$ denotes the MAF of occurrence of at least one event within the range of intensity levels of interest, which is a function of the recurrence law for the seismic source, and $G_{D}(d)=P[D>d]$ is the probability of exceedance of the demand $d$, given the occurrence of an earthquake of any intensity.

Obviously, in order to generate a demand hazard curve, $v_{D}(d)$ must be estimated for different values of the demand, up to very low exceedance probabilities. In this study, the demand hazard curves are estimated via Subset Simulation. The basic idea behind this advanced simulation technique is to express the rare-event probability $G_{D}\left(d_{l}\right)$ in terms of the product of larger conditional probabilities, by introducing intermediate exceedance events corresponding to lower threshold values $d_{1}<d_{2}<\ldots<d_{l}$.

Several improved versions of Subset Simulation have been proposed in the literature, such as Subset Infinity (Au and Patelli 2016), whose algorithm is made available in OpenCOSSAN library (Patelli 2017). However, for the purposes of this work the original version (Au and Beck 2003; Au and Wang 2014) of the method is employed, since improving the efficiency of the simulation approach is out of scope of the paper. This relies on the Markov Chain Monte Carlo algorithm and the MetropolisHastings sampler to efficiently and adaptively generate samples conditional on the intermediate failure regions and thus gradually populate from the frequent to rare event region.

Assuming a fixed value $p_{0}$ for the conditional probabilities of exceedance of the various thresholds, each time a set of $n_{\text {sim }}$ samples is generated through the Metropolis-Hastings algorithm (standard Monte Carlo simulation for the first threshold), and the corresponding demand threshold $d_{i}$ is simply evaluated as the $\left(1-p_{0}\right) n_{s i m}$-th largest value. The exceedance probability of the $i$-th threshold, computed by carrying out $i$-times the product of the same probability $p_{0}$, is $p_{0}{ }^{i}$, for $i=1,2, \ldots, l$, and the lowest obtained value of the failure probability is $p_{0}^{l}$.

The results obtained by Subset Simulation (Au and Beck 2003) are practically unbiased and on average they converge to the reference results furnished by the robust direct Monte Carlo simulation. For this reason, the demand hazard curves evaluated via Subset Simulation can be used as reference solutions against which the estimates obtained through the conditional approach are compared. 
The conditional approach for demand hazard assessment decomposes the estimation of $v_{D}(d)$ into two steps. The first one is the evaluation of the hazard function $v_{I M}(\mathrm{im})$, i.e., the MAF of exceeding the value $\mathrm{im}$ of the intensity measure $I M$. As discussed before, the seismic hazard analysis is not performed by using empirical ground motion prediction equations (GMPEs) but rather via a simulation approach, using a stochastic ground motion model. In particular, the $I M$ hazard curve $v_{I M}$ is built via Subset Simulation, by solving the same problem of Eq. (1) with the $I M$ in place of the generic demand parameter $D$.

Once the seismic hazard has been characterised, the second step of the conditional approach consists in building the probabilistic demand model (Tubaldi et al. 2016; Freddi et al. 2017). This model links the generic demand $D$ with the $I M$ through the function $G_{D \mid I M}(d \mid i m)$, denoting the probability of exceeding the demand value $d$ conditional to the seismic intensity level $i m$. Finally, as a result of the Total Probability Theorem, the mean annual rate of exceedance $v_{D}(d)$ can be estimated by solving the following convolution integral between the seismic hazard function $v_{I M}$ and the conditional demand function $G_{D \mid I M}$ :

$v_{D}(d)=\int_{I M} G_{D \mid I M}(d \mid i m)\left|d v_{I M}\right|$

For the sake of clarity, a flow-chart summarising the main steps of demand hazard estimation according to the $I M$-based approach with stochastic ground motion samples is provided in Fig. 1.

The integral of Eq. (2) can be computed numerically by employing standard integration rules (i.e., rectangle, trapezoidal) or more sophisticated approaches that have been proposed recently (Bradley et al. 2009). In this study, the standard trapezoidal rule is used to solve the integral of Eq. (2), while MSA is employed to build the $G_{D \mid I M}$ function, which requires performing a number of nonlinear dynamic structural analyses at discrete $I M$ levels. On this regard, it is worth noting that the application of Subset Simulation for seismic hazard analysis provides a partitioning of the $I M$ domain into $I M$ intervals with increasing amplitude. This is a consequence of the shape of the hazard curve and of the choice of a fixed value $p_{0}$ for the conditional probabilities of exceedance, resulting in an equal spacing in the logarithmic scale between the MAFs of exceedance of adjacent $I M$ levels. Moreover, Subset Simulation automatically performs hazard disaggregation (Bazzurro and Cornell 1999) in the sense that the seismological features of the earthquake samples generated for the different $I M$ thresholds and exceedance probabilities change coherently with the seismic hazard level. This simplifies the selection of the ground motion records to be used for MSA. In fact, $n_{\text {sim }}$ records are required to perform MSA at each of the $n_{I M} I M$ levels, and these records can be taken from those generated through Subset Simulation at each IM interval. If Subset Simulation is carried out by considering a large number of samples for each $I M$ interval, it is possible to find many records with intensities close to the target ones, and thus record scaling to achieve the target $I M$ can be avoided (as in the present case).

The convolution between hazard and fragility functions is performed by using the same number of $I M$ levels $\left(n_{I M}\right)$ adopted for the hazard curve discretisation, similarly to many probability-based seismic assessment studies (Vamvatsikos and Allin Cornell 2002) (Iervolino et al. 2018) (Scozzese et al. 2018b). Having evaluated the structural response through the $n_{\text {sim }} \cdot n_{I M} \operatorname{simulations,~}$ it is possible to build the demand model $G_{D \mid I M}(\mathrm{~d} \mid \mathrm{im})$ with the so-called "empirical approach", which can be mathematically written as follows (Pinto et al. 2004):

$G_{D \mid I M}(d \mid i m) \cong \frac{1}{n_{\text {sim }}} \sum_{k=1}^{n_{\text {sim }}} I_{k}(d \mid i m)$

where $I_{k}(d \mid \mathrm{im})$ is an indicator function, equal to one if $d_{k}>d$ for the $k$-th record at $I M=i m$ and zero otherwise. Alternatively, the conditional demand model $G_{D \mid I M}(d \mid i m)$ can be estimated via "parametric approach", e.g. assuming a lognormal distribution of the demand value $d$ conditional to the seismic intensity level $\mathrm{im}$. This is a common assumption accepted by the research community (Shome and Cornell 1999; Aslani and Miranda 2005; Bradley et al. 2010) and quite useful for achieving closed-form risk estimates. Alternative distributions of the structural demand have also been proposed in the literature (Romão et al. 2011). Unless stated otherwise, the "empirical approach" is used to estimate the demand model $G_{D \mid I M}(d \mid i m)$ in the following of the paper.

It is noteworthy that another method widely employed in performance-based earthquake engineering (PBEE) for seismic response assessment is incremental dynamic analysis (IDA). The main difference of IDA compared to MSA is that it employs a single ensemble of $n_{\text {sim }}$ records, which are scaled to increasing amplitude levels, generally up to the attainment of collapse condition. Although still widely used, concerns have been raised on IDA by various authors, in particular about the legitimacy of scaling a single set of records over a wide range of IMs (Lin and Baker 2013)(Bradley 2013b)(FEMA 2005).

MSA partially overcomes this problem, although recourse to scaling becomes unavoidable when natural ground motions are used. This drawback may be overcome by employing a conditional mean spectrum method (Baker and Cornell 2006)(Kwong 
and Chopra 2016)(Lin et al. 2013) for record selection. However, it may still be difficult to find records for high intensity levels (corresponding to very large magnitudes) without scaling. The alteration induced by records scaling is widely discussed and analysed in the literature (Der Kiureghian and Fujimura 2009)(Lin et al. 2013)(Jalayer and Beck 2008)(Dávalos and Miranda 2019b).

It is noteworthy that the problem of scaling is overcome by using a stochastic earthquake input, despite other sources of approximation might be introduced by this way.

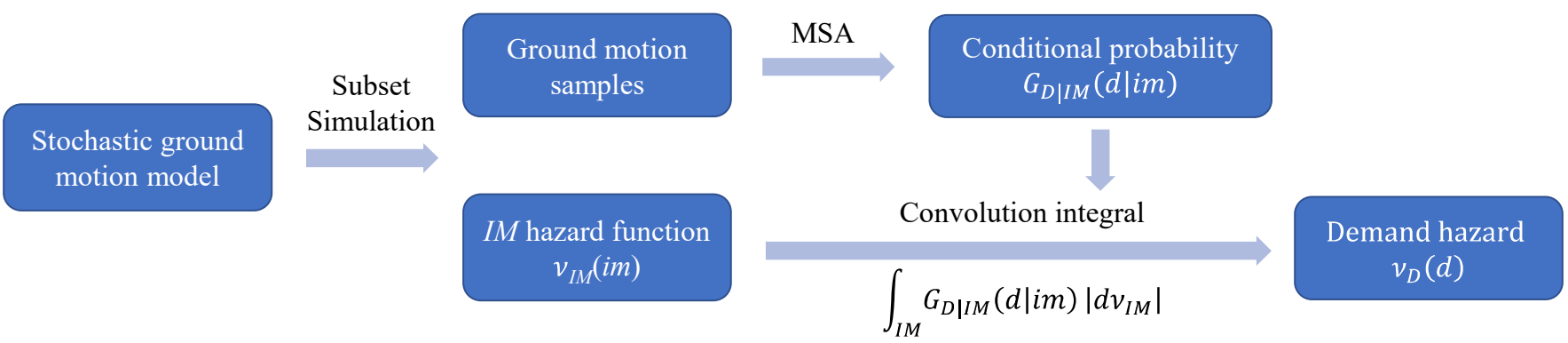

Fig. 1 Flow-chart illustrating the steps for demand hazard estimation via the unconditional approach.

\subsection{Assessment of the conditional approach}

The efficiency and accuracy of the conditional approach may be significantly affected by the setup choices and values assigned to the parameters controlling MSA. These are: the number $n_{I M}$ of $I M$-levels (IM-stripes); the number of ground motion samples per stripe $n_{\text {sim }}$; the way $G_{D \mid I M}$ is estimated (via empirical or parametric approach); the size of the whole $I M$ range investigated, as controlled by the lowest value of $v_{I M}(\mathrm{im})$ attained by the $I M$ hazard curve (hereafter denoted by $\hat{v}$ ).

The influence of these parameters on the efficiency and accuracy of the MSA-based conditional method is assessed in the next sections by analysing first the SDOF system and then the MDOF model.

For each system, a set of EDPs is monitored and the demand hazard curves obtained via conditional method are compared to the reference curves obtained via Subset Simulation. The sensitivity to the various controlling parameters listed above is carried out by starting from a default setting of the conditional method, and by modifying one single parameter at a time. Multiple independent runs are performed for both the conditional and the unconditional approaches.

For each EDP the results are presented in terms of both average demand hazard curves and coefficients of variation (COVs). The comparison between the average hazard curves allows assessing the potential bias of the conditional approach.

In particular, by introducing the inverse function of $v_{D}$, denoted as $d(v)$, this bias can be quantified through the normalized measure of the difference between the reference and the conditional mean demand functions:

$e_{D}(v)=100 \frac{d_{M S A}(v)-d_{R e f}(v)}{d_{R e f}(v)}$

where $d_{R e f}(v)$ refers to the average demand estimated via Subset Simulation, $d_{M S A}(v)$ represents the same quantity estimated via MSA-based conditional approach. The normalised differences of Eq. (4) are evaluated at fixed values of $v$, namely $10^{-2}, 10^{-}$ ${ }^{3}, 10^{-4}, 10^{-5}, 10^{-6} 1$ year. High absolute values of $e_{D}$ denote significant bias in the estimates of the conditional approach, and positive sign indicates that the conditional method yields an overestimation of the demand compared to the unconditional one. Obviously, the bias cannot be eliminated by increasing the number of simulations or by changing the values of the other setup parameters controlling the probabilistic approach. Besides the normalized measures above and as synthetic descriptor of the bias, the root mean square errors (RMSEs) are also provided, based on the estimates of $e_{D}$ at the aforesaid five MAF levels:

RMSE $=100 \sqrt{\frac{\sum_{i=1}^{5}\left[e_{D i}(v)\right]^{2}}{5}}$

The statistical precision of the conditional approach is quantified by the values of two different COVs: the COVs of the demand estimates $d(v)$ at fixed MAF levels $v$ (namely $10^{-2}, 10^{-3}, 10^{-4}, 10^{-5}, 10^{-6} 1$ year), and the COVs of the MAF of exceedance $v_{D}(d)$ at fixed demand levels. Obviously, higher values of the COVs obtained via the conditional method compared to those obtained via the unconditional approach denote less precision of the method or, in other words, a less confident estimate of the demand hazard curve for the EDP at hand. 


\section{Case study description}

This section describes the case studies considered for evaluating the effectiveness of MSA and for assessing the influence of the various setup parameters on the efficiency and accuracy of the method. Subsection 3.1 provides details on the seismic scenario and stochastic ground motion model considered in the study, whereas Subsection 3.2 describes the structural system properties and relevant monitored EDPs.

\subsection{Seismic scenario and stochastic ground motion model}

Similarly to (Au and Beck 2003; Jalayer and Beck 2008; Vetter and Taflanidis 2012), the seismic scenario is described by a single source model, characterized by two main random seismological parameters, namely the moment magnitude $M$, and the epicentral distance $R$. A Gutenberg-Richter recurrence law (Kramer 2003) (Eq. 6) is used to describe the magnitude-frequency relationship of the seismic source:

$v_{M}(m)=10^{(a-b m)}$.

in which the parameter $a$ accounts for the mean number of earthquakes expected from the seismic source, while the parameter $b$ is a regional seismicity factor governing the proportion of small to large earthquakes. The assumed recurrence law, bounded within the range of magnitudes of interest $\left[m_{0}, m_{\max }\right]$, leads to the following probability density function (PDF) of the moment magnitude (Kramer 2003; Au and Beck 2003):

$f_{M}(m)=\beta \frac{e^{-\beta\left(m-m_{0}\right)}}{1-e^{-\beta\left(m_{\max }-m_{0}\right)}}$

being $\beta=b^{*} \log _{e}(10), m_{0}$ the magnitude value below which non-significant effects are expected on the structures, and $m_{\max }$ the physical upper bound of the magnitudes expected from the source. In this application, as well as in (Au and Beck 2003), it is assumed $m_{0}=5, m_{\max }=8, a=4.5$ and $b=1$. With these parameters, the annual rate of exceedance $\bar{v}$ of earthquakes of any magnitude between $m_{0}$ and $m_{\max }$ is equal to 0.3161 /year.

The epicentral distance is modelled according to the following PDF:

$f_{R}(r)=\left\{\begin{array}{l}\frac{2 r}{r_{\text {max }}} \text { if } r<r_{\text {max }} \\ 0 \quad \text { otherwise }\end{array}\right.$

which is obtained under the hypothesis that the source produces random earthquakes with equal likelihood anywhere within a distance from the site $r_{\max }=50 \mathrm{~km}$, beyond which the seismic effects are assumed to become negligible (Au and Beck 2003). The soil condition is described by a deterministic value of the shear-wave velocity parameter $V_{S 30}=310 \mathrm{~m} / \mathrm{s}$, representative of average soil condition (Boore and Joyner 1997).

The Atkinson-Silva (Atkinson and Silva 2000) source-based ground motion model is used to describe the attenuation from the source to the building site. This model, combined with the stochastic point source simulation method of (Boore 2003), is employed to generate ground motion samples conditional to the samples of $M, R$. Fig. 2 illustrates the ground motion total radiation spectrum $A(\omega)$ (i.e., the Fourier spectrum), and the time-envelope function $e(t)$, obtained for different earthquake moment magnitudes $m(5,6.5,8)$ and a fixed epicentral distance $r=20 \mathrm{~km}$. The ground motions record-to-record variability is simulated through the following two quantities: a Gaussian white noise process and a lognormal scale factor of the radiation spectrum. In particular, for each earthquake sample a Gaussian white noise signal is generated and, after being windowed through the envelope-functions $e(t)$ (Fig. 2b), its normalized frequency spectrum is applied to the target radiation spectrum (Fig. 2a), thus providing the variability of the energy content within the frequency domain. Such variability is further amplified by the lognormally-distributed multiplicative factor of the radiation spectra, $\varepsilon_{m o d}$, characterised by a unitary median value and a standard deviation $\sigma_{\mathrm{ln}}=0.5$, as proposed by (Jalayer and Beck 2008). The resulting overall variability provided by the model is shown in Fig. 3a, in which the spectra of three earthquake samples corresponding to the same pair of magnitude and distance (i.e., $m=6.5$ and $r=20 \mathrm{~km}$ ) are depicted in different colours. It can be observed how the Fourier spectral amplitudes differ sample-by-sample, with peaks randomly distributed over the frequencies, although on average the trends are fully defined once the input parameters are fixed $\left(M, R, V_{S 30}, \varepsilon_{m o d}\right)$. For the sake of completeness, the acceleration time series corresponding to the three aforesaid spectra are also plotted in Fig. $3 \mathrm{~b}$.

Once the seismic scenario is defined, the hazard curve can be built by applying Subset Simulation. It is recalled that the choice of the $I M$ affects the quality of the demand estimates with the conditional approach, in terms of accuracy (or bias, referring to the closeness of the estimate to the reference value) and precision (referring to the variability of the estimates, hence related to 
the demand dispersion for a given $I M$ ). While the accuracy is more related to the sufficiency of the $I M$, the precision depends on its efficiency (Luco and Cornell 2007). In this study, the spectral acceleration at the fundamental period of the system, $S_{a}(T)$, is used as $I M$.

Fig. 4 plots the $I M$ hazard curves obtained from multiple runs of Subset Simulation (20 independent simulations) and the corresponding average curve. The computational effort required to build the hazard curve is very low, since structural analyses must be performed on a damped linear elastic SDOF system (with period $T=1.0 \mathrm{~s}$ and damping ratio $\xi=0.02$ ) by monitoring only a single demand parameter (i.e., $S_{a}(T)$ ). The value of the period used for conditioning the $I M$ is $1.0 \mathrm{~s}$, as well as the value of the fundamental period of the MDOF model.

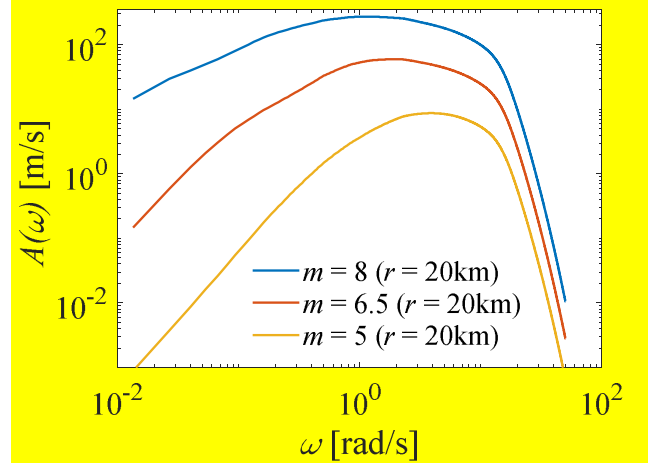

a)

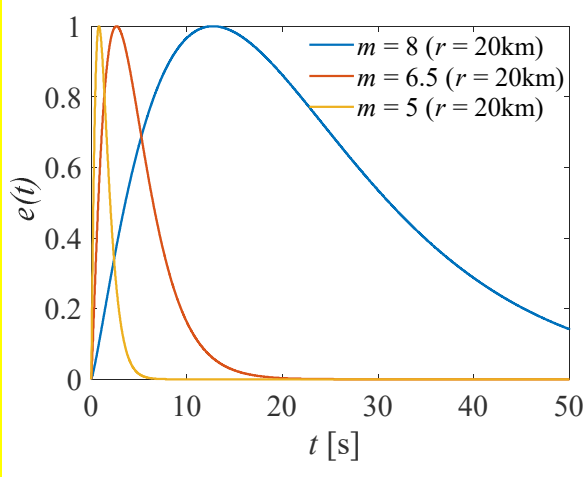

b)

Fig. 2 a) Radiation Fourier spectra and b) time-envelope functions for $r=20 \mathrm{~km}$ and different $M$ values.

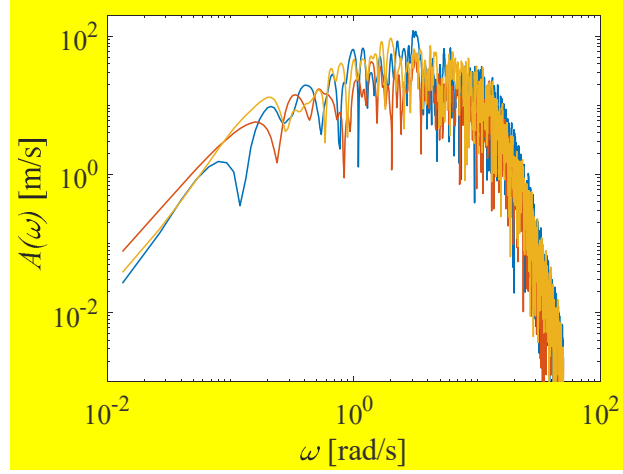

a)

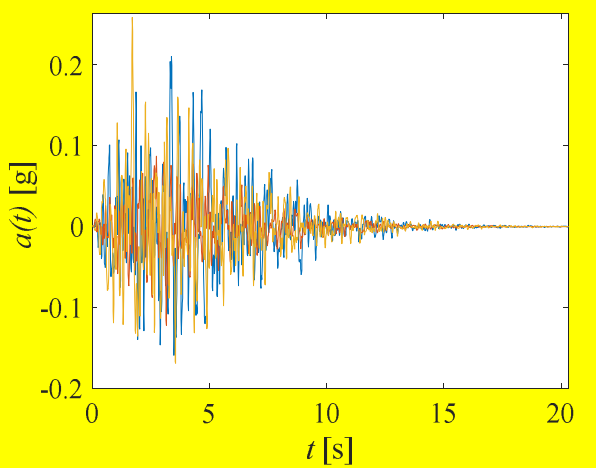

b)

Fig. 3 a) Radiation Fourier spectra and b) acceleration time series for three different stochastic simlations with $m=6.5$ and $r=20 \mathrm{~km}$.

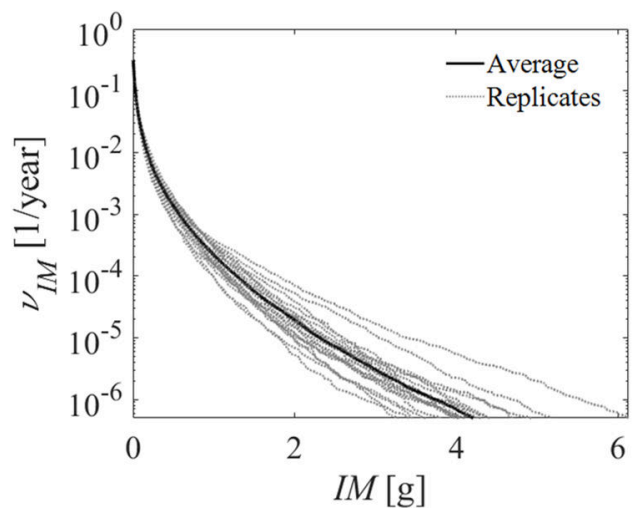

Fig. 4 Hazard curves for $S_{a}(T)$ corresponding to multiple runs of Subset Simulation and average hazard curve.

\subsection{Structural system properties}

The structural system considered in this study consists in a 3-storey steel moment-resisting frame building, designed within the SAC Phase II Steel Project, and widely used as benchmark structure in several other works concerning structural response control (Gupta and Krawinkler 1999; Barroso and Winterstein 2002; Ohtori et al. 2004; Dall'Asta et al. 2016; Scozzese et al. 2019). The frame was designed for gravity, wind, and seismic loads in order to conform to local code requirements in Los Angeles, California region. As shown in Fig. 5, the whole structural system consists of perimeter moment-resisting frames and internal gravity frames 
with shear connections, while the structural model for analysis purposes is a two-dimensional frame representing one half of the structure in the north-south direction. The main geometrical details and the size of the steel members (wide-flange sections are used for both columns and beams) are shown in Fig. 5. Further details concerning the structural geometry and loads can be found in (Ohtori et al. 2004).

The finite element model of the system is developed in OpenSees (McKenna 1997)(Mazzoni et al. 2006) following the approach described in (Dall'Asta et al. 2016) and briefly recalled below. A distributed plasticity approach is adopted (Yu et al. 2013; Seo et al. 2014; Scozzese et al. 2018a), with nonlinear force-based elements and fiber sections with Steel02 uniaxial material (elastoplastic constitutive law with smooth elastic-to-plastic transition). An elastic fictitious P-delta column is introduced to account for the nonlinear geometrical effects induced by the relevant vertical loads, including those carried by the inner gravity frames that are not explicitly modelled. A corotational transformation is used to describe the large-displacement small strain problem. The strength and deformability of panel zones are neglected. A Rayleigh modelling of the elastic damping properties is used, by assigning a $2 \%$ damping ratio at the first two vibration modes.

Periods of the first three vibration modes $T_{\mathrm{i}}$ and related percentages of participating mass $M_{p, \mathrm{i}}$ are summarised in Table 2 . The capacity curve of the frame, obtained from a pushover analysis performed by considering a lateral load patter proportional to the first modal shape, is shown in Fig. 6 in terms of base shear $V_{b}$ normalized by the self-weight $W$ and roof drift angle (i.e., the top displacement divided by the height). Because of the adopted modelling strategy, no sign of softening behaviour is observed, and thus the collapse can only be conventionally defined as the attainment of 0.1 limit drift value (maximum abscissa value shown for the capacity curve in Fig. 6). More refined modelling approaches might be adopted to account for both strength and stiffness degradation of the structural elements (Lignos and Krawinkler 2010) and thus explicitly simulate the global collapse of the building, however this is out of the scope of the present work.

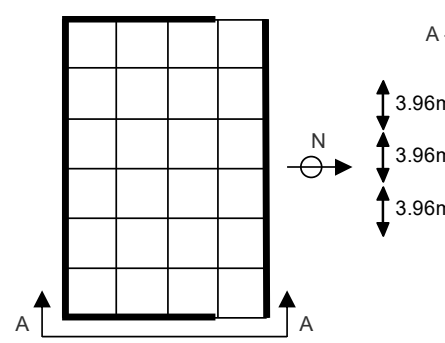

a)

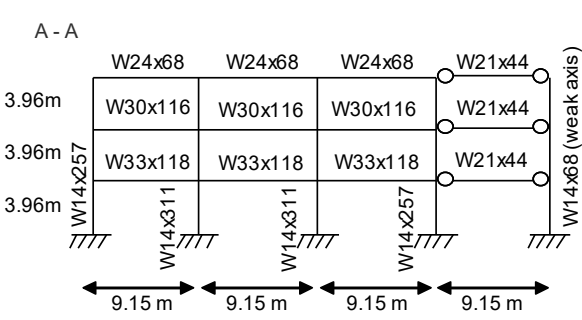

b)

Fig. 5 a) Plan (thick lines highlight moment-resisting frames) and b) elevation of the 3-storey steel frame.

Table 2 Vibration periods for the 3-storey steel moment-resisting frame

\begin{tabular}{ccc}
\hline Mode & $T_{\mathrm{i}}[\mathrm{s}]$ & $M_{p, \mathrm{i}}[\%]$ \\
\hline 1 & 0.995 & 82.717 \\
2 & 0.325 & 13.571 \\
3 & 0.173 & 3.708 \\
\hline
\end{tabular}

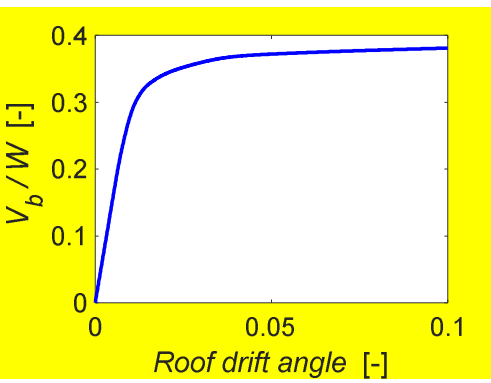

Fig. 6 Capacity curve in terms of base shear $V_{b}$ normalized by the self-weight $W$ and roof drift angle (top displacement divided the height).

In order to investigate wide ranges of choices in the setup of MSA, a simplified model of the structure is considered. This consists in a nonlinear SDOF system with fundamental period $T_{1}$, yielding force per unit mass $V_{y} / m$, post-elastic stiffness ratio $k_{p} / k$, and the damping ratio $\xi$. The values assumed for these properties, reported in Table 3, are chosen such that the dynamic behaviour of the SDOF system represents that of the steel moment resisting building described above. More precisely, the SDOF model, 
built in OpenSees (McKenna 1997)(Mazzoni et al. 2006) using the bilinear Steel02 constitutive law (with smooth transition from the elastic to the inelastic field), has a response under lateral forces consistent with the one of the MDOF system.

Table 3 Properties of the SDOF system

\begin{tabular}{cccc}
\hline$T$ & $V_{y} / m$ & $k_{p} / k$ & $\xi$ \\
{$[\mathrm{s}]$} & {$\left[\mathrm{m} / \mathrm{s}^{2}\right]$} & {$[-]$} & {$[-]$} \\
\hline 1.00 & 4.21 & 0.01 & 0.02 \\
\hline
\end{tabular}

\section{Parametric study results for nonlinear SDOF system}

In this section, an extensive parametric study is carried out on a nonlinear SDOF system to assess the influence of the various parameters governing the MSA-based conditional method. The reference solution obtained via Subset Simulation (Au and Beck 2003 ) is presented and discussed in Subsection 4.1. In Subsection 4.2, the solution obtained with the conditional approach is presented. Successively, the parametric study is performed, by considering the default setting of the method and by changing one single parameter at a time. The obtained results are illustrated in Subsections 4.3-4.6. Three demand parameters are considered to describe the seismic performance of the SDOF system: the maximum displacement demand $u$, providing indirect information on the structural damage; the maximum absolute force per unit mass $V_{b} / m$, which also corresponds to the maximum absolute acceleration and thus provides indirect information on the response of (acceleration-sensitive) non-structural components and foundations; the maximum residual displacement $u_{\text {res }}$, a parameter providing insights on post-earthquake retrofit costs and interventions (Ruiz-García and Miranda 2006).

\subsection{Risk estimation with Subset Simulation: reference solution}

The reference solutions in terms of demand hazard curves for the EDPs of interest are evaluated via Subset Simulation. The number $l$ of simulation levels, the actual value of $p_{0}$, as well as the number of simulations per level $n_{\text {sim }}$ must be set based on the specific reliability problem to be solved. In the present study, $p_{0}=10 \%, l=7$, and $n_{\text {sim }}=500$ are assumed to achieve a reliable estimate of the risk up to very low MAF of exceedances, in the range $10^{-5}-10^{-6}$. This is the range of values that according to Eurocode 0 should be targeted in designing a structure (Eurocode 0 2002).

It is noteworthy that the values of $p_{0}$ and $l$ assumed here to derive the reference demand hazard curves are different from those considered in the next Section, where Subset Simulation is used within the framework of the conditional approach to build the $I M$ hazard curves. According to the current setup, 3500 analyses are required by a single Subset Simulation. A total of 20 independent runs of Subset Simulation are carried out for each demand parameter. Although significant savings in terms of computational cost are achieved with respect to classic Monte Carlo simulation, the number of analyses required in this work is still quite high, even for a SDOF system.

The demand hazard curves for the monitored EDPs are plotted in Fig. 7. The curves obtained for the various independent runs of Subset Simulation are shown with grey dotted lines, and the corresponding average demand hazard curves are shown with black solid lines. As expected, the various EDPs exhibit different trends of the demand hazard curves. In particular, the maximum normalised force (Fig. 7b) has a sharp change of slope in correspondence of the yield point. This is due to the low hardening behaviour of the system following yielding, which limits the increase of forces and thus of the absolute accelerations.

The curve of the residual displacements (Fig. 7c) follows a different trend, and non-negligible values, higher than $10^{-3} \mathrm{~m}$, are attained for $v_{D}<0.0021$ year. The presence of non-null residual displacements (though very small) for $v_{D} \geq 0.0021 /$ year is due to the constitutive law considered for the SDOF system, with a smooth transition from the elastic to the inelastic field.

The confidence of the estimates obtained via Subset Simulation is quantified by the COVs of $v_{D}(d)$ and $d(v)$, shown in Fig. 8 . As a general result, the COVs of $v_{D}(d)$ span from 0.2 to 0.8 , with higher values corresponding to lower MAF of exceedances. The COVs of $d(v)$ are always lower than 0.4 , and their trends of variation with the MAF are irregular and strongly depend on both the shape of the hazard curve of the specific demand parameter $D$, and on $D$ itself. For instance, the COVs of $d(v)$ for $u_{r e s}$ are higher than for the other demand parameters.

Both the mean demand hazard curves and the COVs given here are assumed as reference solutions and they are used to test the results obtained with different setups of the MSA-based conditional approach. 


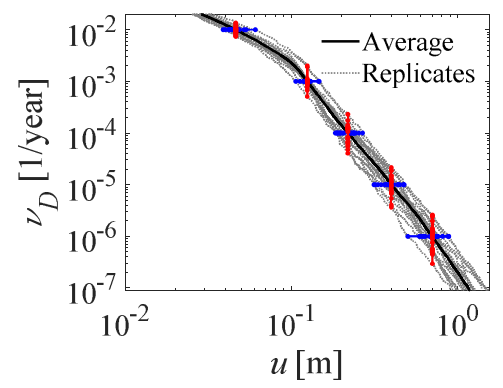

a)

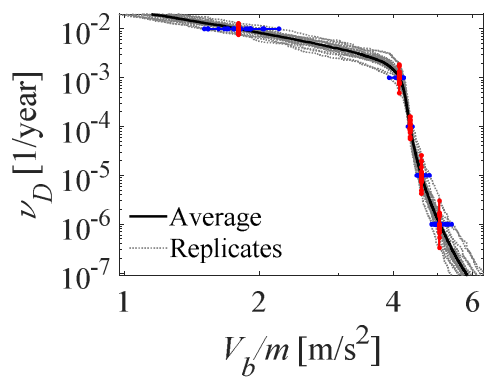

b)

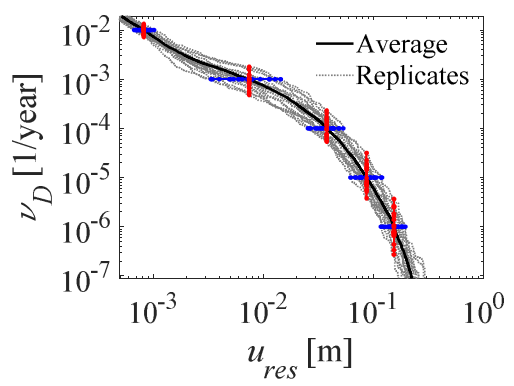

c)

Fig. 7 Reference demand hazard curves obtained averaging 20 independent runs of Subset Simulation.

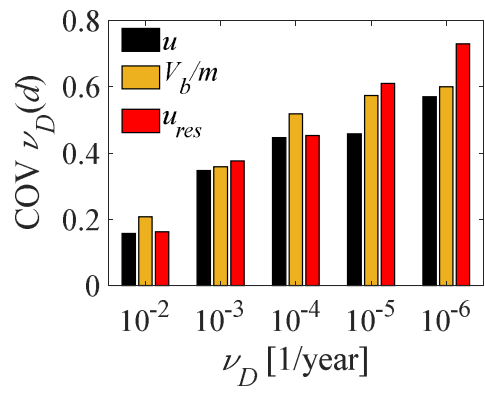

a)

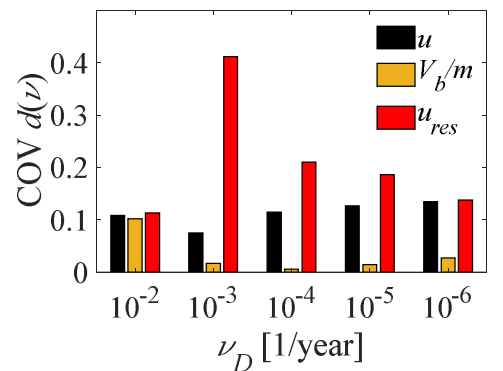

b)

Fig. 8 Reference COVs of a) $\boldsymbol{v}_{\boldsymbol{D}}(d)$ and b) $d(\boldsymbol{v})$ from Subset Simulation for different EDPs.

\subsection{MSA-based conditional solution: controlling parameters and reference setup}

This subsection evaluates the efficiency and accuracy of the MSA-based conditional method. The results presented here are obtained by using the "default setting" of the conditional method, which consists of:

- IM hazard curves bounded in a range of MAF values between $\overline{\boldsymbol{v}}=0.3161 /$ year and $\hat{\boldsymbol{v}}=3 \cdot 10^{-7} 1 /$ year;

- IM hazard curves discretised into a number of intervals equal to 20, which corresponds to a number of levels (IM-stripes) of analysis equal to $\mathrm{n}_{\mathrm{IM}}=21$;

- a different set of $\mathrm{n}_{\text {sim }}=20$ stochastic acceleration time series is selected at each IM-level, reflecting the change of spectral content and duration with the seismic intensity level (see Appendix A for details on the IM-hazard curve generation via Subset Simulation), for a total of 420 nonlinear dynamic analysis per each MSA simulation.

It is worth noting how the whole number of simulations is slightly higher compared to those usually carried out in practical applications of probability-based seismic assessment (Vamvatsikos and Allin Cornell 2002) (Iervolino et al. 2018) (Bradley 2013a)(Scozzese et al. 2018b). In these applications, indeed, 200 simulations or less are carried out, without providing any explanation about the choice and the level of accuracy achieved in the risk estimation. A schematic illustration of the main parameters governing the problem (listed above) is provided on the $I M$ hazard curve of Fig. 9a. The results obtained by modifying the default setting are illustrated in the next subsections.

In order to assess the accuracy of the conditional approach, 20 independent conditional analyses are carried out, each performed by using a different $I M$ hazard curves (an example of $I M$ hazard curve from a single replicate among the 20 ones is shown in Fig. $9 b$ ) and a different set of earthquake samples. The demand hazard curves resulting from the analyses are plotted in Fig. 10 in grey dotted lines, together with the mean demand hazard curve (red dashed line) and the reference solution obtained by averaging the results obtained via Subset Simulation (presented in the previous subsection).

The bias of the conditional approach is quantified numerically through the error $e_{D}$ (presented before, see Eq. (4)), providing a normalized measure of the distance between the demand hazard curves according to the two approaches at fixed values of $v_{D}$ (namely $10^{-2}, 10^{-3}, 10^{-4}, 10^{-5}, 10^{-6} 1$ year). The percentage error values $e_{D}$ are collected in Table 4 , together with the root mean square error (RMSE) values (see Eq. (5)). In general, the values of $e_{D}$ in Table 4 are always lower than 5\% for all the EDPs and MAF levels monitored, with the exception of the error for the residual displacement $u_{\text {res }}$ at $v_{D}=10^{-2} 1 /$ year, which is equal to $11.53 \%$. The low error values demonstrate the unbiasedness of the conditional estimator with the adopted analysis setting, which can also be appreciated in Fig. 10, showing that the mean demand hazard curves according to the two approaches are practically coincident. 
The statistical precision of the conditional method is assessed through the COVs of $v_{D}(d)$ and $d(v)$, whose values illustrated in Fig. 11 have been obtained from the 20 independent runs of the conditional approach, for all the demand parameters considered. The dispersion of the results is comparable to that of the results obtained with the unconditional approach for $v_{D}$ values higher than $10^{-4} 1$ year. For lower values of $v_{D}$, the COVs of the conditional approach are higher than the reference ones and increase for decreasing $v_{D}$ values. In particular, the COVs of $v_{D}(d)$ increase for decreasing MAFs of exceedance as a consequence of the higher dispersion of the response contributing to the exceedance of the threshold $d$. On the other hand, the COVs of $d(v)$ do not follow a regular trend but they are strongly influenced by the specific demand parameter $D$ analysed. Apart from the differences in value, the trends of the COVs for the conditional approach are in agreement with those obtained with the unconditional approach. In particular, the trends of the COVs of $d(v)$ for the normalised force (see Fig. 11b, bottom chart) show a sudden reduction of the demand dispersion following the system yielding. A rapid increase of the residual displacements is observed once this condition has been attained.

To summarize the obtained results, the default setting of the parameters controlling the numerical solution with MSA provides demand hazard curves that are on average unbiased, with a precision globally comparable to that of the unconditional approach. In light of this, the method setup presented here can be assumed as the reference one for what concerns the conditional solution. In the next subsections, the influence of the various parameters controlling the accuracy of the conditional approach is assessed through an extensive parametric study, in which the default setting presented here is changed by varying one single controlling parameter at a time. In particular, the parameters governing the discretisation of the hazard curve (i.e., the effect of $n_{I M}$ ), the number of analyses per IM-stripe (i.e., the effect of $n_{\text {sim }}$ ) and the $I M$ hazard curve truncation (effect of $\hat{v}$ ) are varied in the next subsections of the paper to investigate the convergence properties of the solution with respect to these parameters.

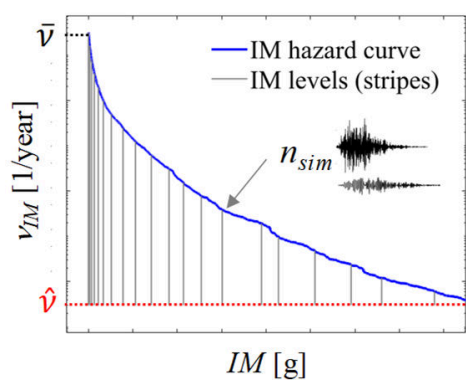

a)

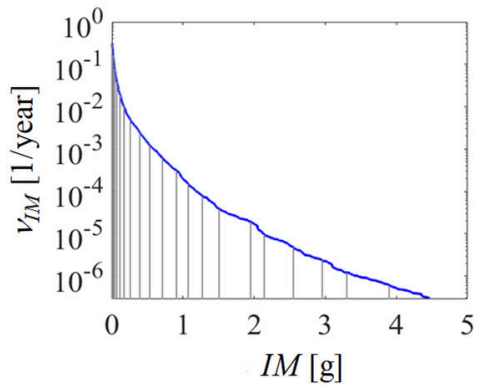

b)

Fig. $9 I M$ hazard curve: a) schematic representation of the main parameters; b) single replicate with $n_{I M}=21$ (i.e., $20 I M$ intervals).

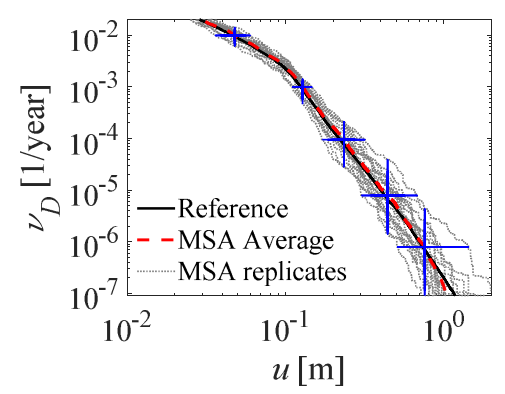

a)

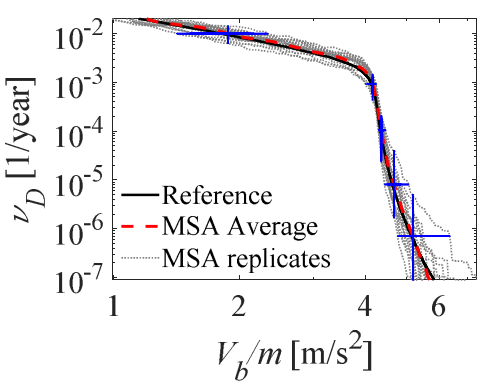

b)

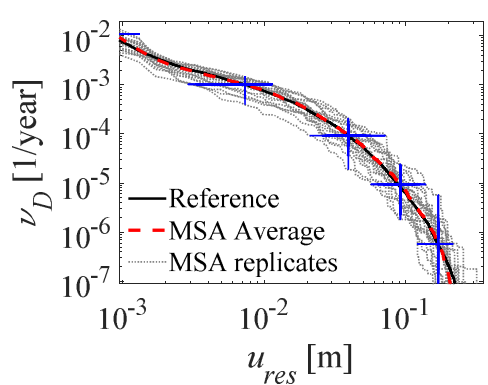

c)

Fig. 10 Conditional simulation replicates and corresponding average curves compared to the reference solution provided by Subset Simulation, for each EDP. Cross marks highlight the points at which the COVs of $v_{D}$ (vertical) and of $d$ (horizontal) are computed. 


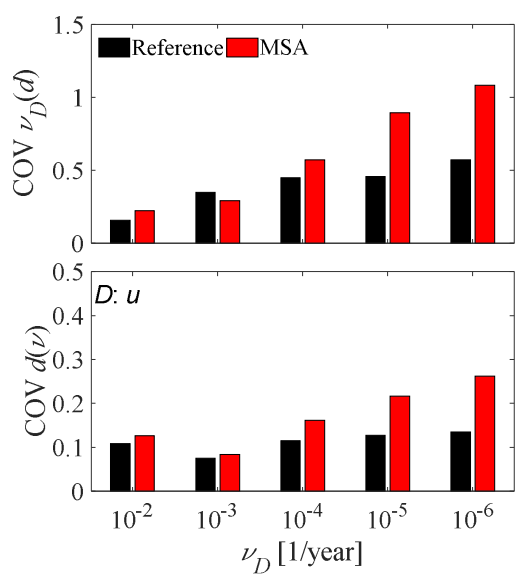

a)
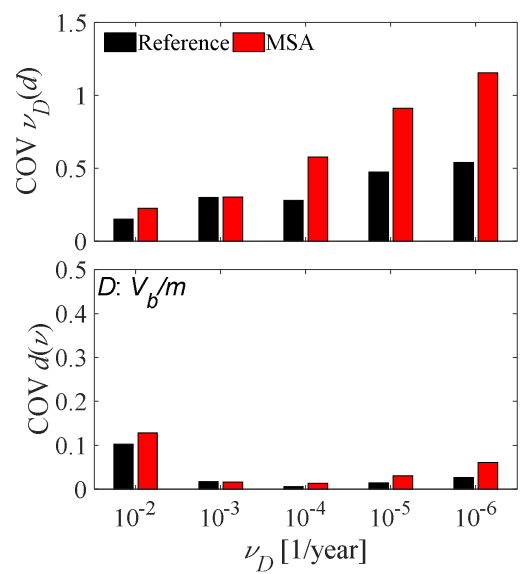

b)

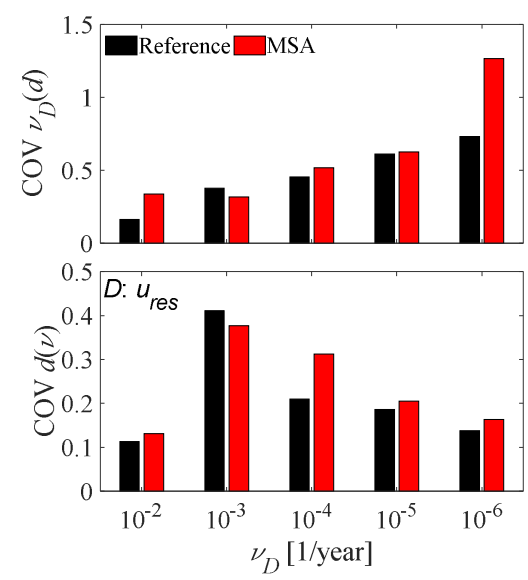

c)

Fig. 11 Comparison of the COVs of the MSA-based conditional solution with the reference values from Subset Simulation. COVs of $\boldsymbol{v}_{\boldsymbol{D}}(d)$ and $d(v)$ for the demand parameters: a) $u$, b) $V_{b} / \mathrm{m}$, c) $u_{\text {res. }}$.

Table 4 Estimation errors $e_{D}$ at different MAFs.

\begin{tabular}{l|lllll|l}
\hline & \multicolumn{5}{|c|}{$e_{D}[\%]$} & \\
\hline$D$ & $v_{D}=10^{-2}$ & $v_{D}=10^{-3}$ & $v_{D}=10^{-4}$ & $v_{D}=10^{-5}$ & $v_{D}=10^{-6}$ & RMSE [\%] \\
\hline$u$ & 2.871 & 3.082 & 4.920 & 3.481 & 1.321 & 7.472 \\
$V_{b} / m$ & 4.200 & 0.702 & 0.494 & 0.740 & 1.042 & 4.471 \\
$u_{\text {res }}$ & 11.532 & -0.631 & 1.930 & 3.912 & 2.270 & 12.553 \\
\hline
\end{tabular}

\subsection{Effect of the $I M$ discretization}

This subsection examines how the $I M$ discretization, and thus the number $n_{I M}$ of $I M$ levels at which the analyses are performed, affects the demand hazard curve estimation for the different EDPs. The IM-curves with the default cut-off value of the MAF at $\hat{v}=3 \cdot 10^{-7}\left(\approx 10^{-7}\right)$ are used. This time, two further discretisation modes are tested, corresponding to $n_{I M}=6$ (Fig. 12a) and $n_{I M}=$ 11 (Fig. 12b) $I M$ levels, in addition to the one already investigated in the previous section $\left(n_{I M}=21\right.$, Fig. $\left.12 \mathrm{c}\right)$. To assess the influence of the $I M$ discretization, the rest of the settings controlling the application of the conditional approach are kept fixed. The average of the 20 demand hazard curves resulting from the conditional approach is plotted, for each EDP, by coloured hatched lines in Fig. 13; the reference average solution provided by Subset Simulation is also shown with a black solid line. The deviation between the conditional and the reference solutions is measured by the errors reported in Table 5 , together with the overall RMSEs.

According to the results shown in Fig. 13 and the values collected in Table 5, the discretization with only 6 IM levels (orange dashed lines) results in some level of bias in the demand hazard curves for all the monitored EDPs. This is quantified by the high values of the estimation errors of Table 5, showing that the demand is almost always overestimated by more than $80 \%$.

With 11 IM levels (red dashed lines), the accuracy of the conditional approach starts improving notably, with the risk of exceedance generally overestimated by $20 \%-30 \%$. Significant errors are still observed at higher rates of exceedance. It is worth noting that the estimation errors have generally a positive sign, and moreover, a poorer $I M$ hazard curve discretisation leads to higher levels of seismic risk overestimation.

For what concerns the effect of the number of $I M$ levels on the results dispersion, a comparison in terms of COVs of $v_{D}(d)$ and $d(v)$ is presented in Fig. 14 for all the demand parameters. In most of the cases, the COVs reduce by increasing the number of $I M$ levels, although few exceptions can be found, in particular regarding the COVs from the analyses on 6 IMs (orange bars in Fig. 14). By increasing the $I M$ discretisation, the COVs become closer to those of the reference setting $\left(n_{I M}=21\right)$, with a rate of convergence particularly high by passing from 6 to $11 \mathrm{IM}$ levels, and a reduced rate by passing from 11 to $21 \mathrm{IMs}$. 


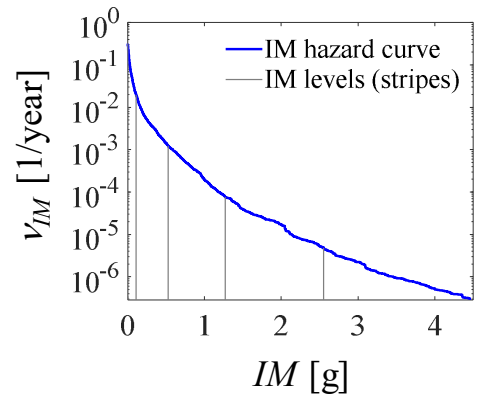

a)

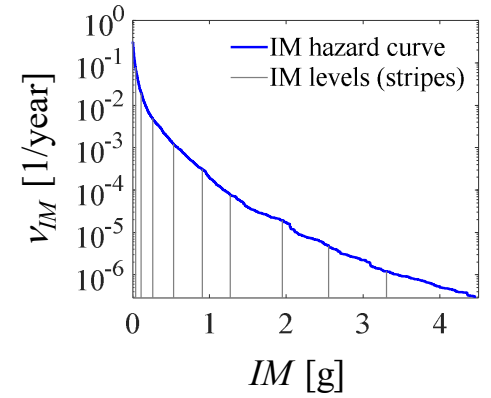

b)

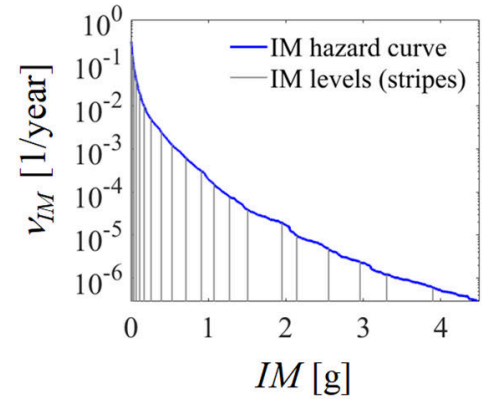

c)

Fig. $12 I M$ hazard curve discretised in: a) 6, b) 11 and c) $21 I M$ levels.

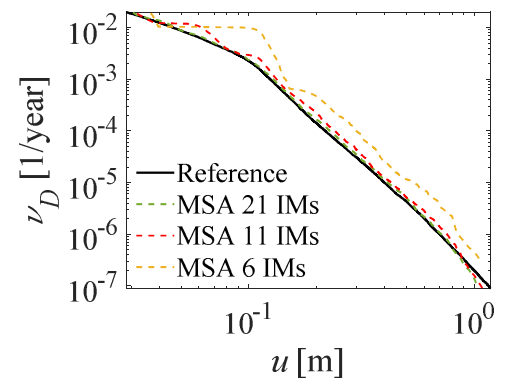

a)

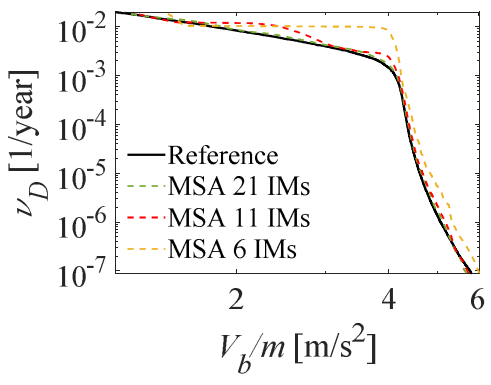

b)

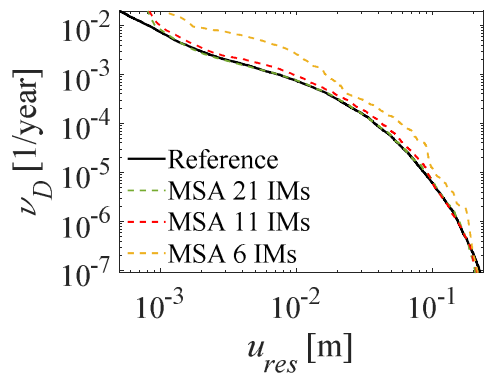

c)
452 453

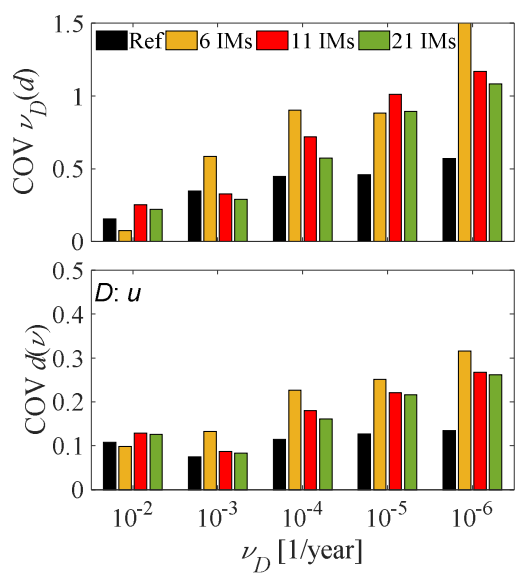

a)
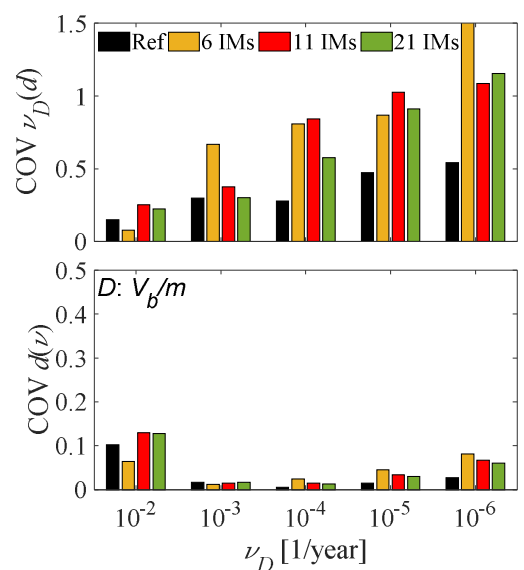

b)
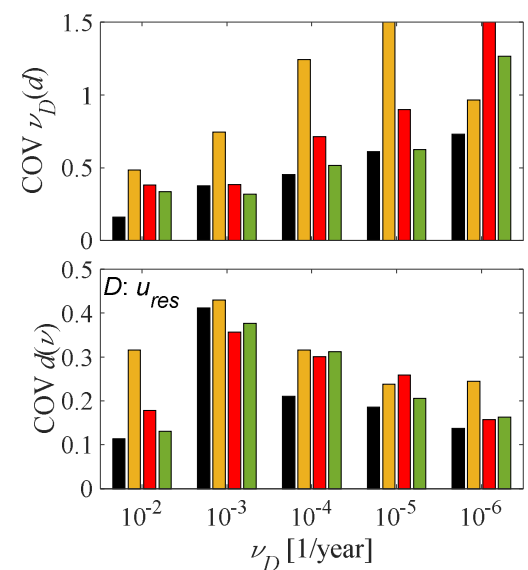

c)
454 455 456

Fig. 14 Comparison of the COVs of the conditional solution with the reference values from Subset Simulation. COVs of $v_{D}(d)$ and $d(v)$ for the demand parameters: a) $u$, b) $V_{b} / \mathrm{m}$, c) $u_{\text {res }}$.

Table 5 Estimation errors $e_{D}$ at different MAFs.

\begin{tabular}{l|l|ll|l|l|l|l}
\hline & & \multicolumn{5}{|c|}{$e_{D}[\%]$} & \\
\cline { 3 - 8 }$D$ & Approach & $v_{D}=10^{-2}$ & $v_{D}=10^{-3}$ & $v_{D}=10^{-4}$ & $v_{D}=10^{-5}$ & $v_{D}=10^{-6}$ & RMSE [\%] \\
\hline \multirow{4}{*}{$u$} & $6 \mathrm{IMs}$ & 109.219 & 16.730 & 36.862 & 33.727 & 19.880 & 122.883 \\
& $11 \mathrm{IMs}$ & 34.190 & 6.676 & 10.466 & 7.763 & 7.511 & 37.944 \\
& $21 \mathrm{IMs}$ & 2.871 & 3.084 & 4.922 & 3.475 & 1.321 & 7.470 \\
\hline \multirow{4}{*}{$V_{b} / m$} & $6 \mathrm{IMs}$ & 93.918 & 2.812 & 2.801 & 4.797 & 5.486 & 94.284 \\
& $11 \mathrm{IMs}$ & 36.024 & 1.484 & 0.916 & 1.248 & 2.273 & 36.159 \\
& $21 \mathrm{IMs}$ & 4.199 & 0.701 & 0.493 & 0.739 & 1.038 & 4.471 \\
\hline \multirow{4}{*}{$u_{\text {res }}$} & $6 \mathrm{IMs}$ & 108.051 & 120.817 & 55.463 & 20.225 & 19.535 & 173.605 \\
& $11 \mathrm{IMs}$ & 21.410 & 26.993 & 12.800 & 5.342 & -1.526 & 37.171 \\
& $21 \mathrm{IMs}$ & 11.527 & -0.632 & 1.927 & 3.910 & 2.269 & 12.546 \\
\hline
\end{tabular}




\subsection{Effect of the number of samples per IM stripe}

This subsection examines how the number of structural analyses performed at each $I M$ level affects the demand hazard curve estimation. For this purpose, the $I M$-curves with $\hat{v} \approx 3 \cdot 10^{-7}$ discretized in $21 I M$ levels are used (default setting), whereas the number of analyses per $I M$ level (i.e., per IM-stripe) is varied as follows: 1 single analysis (for a total amount of $1 \times 21=21$ simulations), 5 analyses (for a total amount of $5 \times 21=105$ simulations), 10 analyses ( $10 \times 21=210$ simulations), 20 analyses ( $20 \times 21=420$ simulations, which corresponds to the default setting of the conditional solution), and 30 analyses $(30 \times 21=630$ simulations). As in the previous investigations, 20 independent runs of MSA analysis are carried out for each case to assess the statistical precision of the method. A graphical comparison between the average demand hazard curves form MSA and the reference method is provided in Fig. 15, whereas Table 6 reports the values of $e_{D}$ at different MAF levels.

The results show that a single sample per IM-stripe (grey dashed lines) might be not sufficient to estimate accurately the demand hazard for values of the MAF of exceedance lower than $10^{-4}$, and this is because the record-to-record variability can't be properly accounted. However, it is worth noting that, the mean demand hazard curve of the EDP maximum displacement $u$ is correctly estimated even with one single analysis per $I M$, as long as the system response is essentially elastic. This is due to the high efficiency of the conditional parameter $(I M)$ in describing the response in terms of the demand parameter $u$. In fact, the analysed system consists in a nonlinear SDOF system with period 1.0s, and hence the conditioning IM and the demand parameter are coincident until the yielding displacement is attained (on average for $v_{D} \approx 10^{-3}$ ). Once the system experiences significant inelastic deformations, the $I M$ becomes less efficient, and this results in a strong bias of the average demand curve of $u$ for $v_{D}<10^{-3}$. It is sufficient to increase to 5 (light blue dashed lines) the number of analyses per $I M$ level to improve the accuracy of the estimator, with a reduction of more than $10 \%$ on the RMSE values (Table 6 ) with respect to the previous case with a single analysis per $I M$ level.

With 10 samples per level (green dashed lines), the accuracy of the conditional approach increases notably: the RMSE values are at least halved compared to the case of 5 analyses per $I M$ level.

With 20 samples per level (red dashed lines) the match between the MSA curves and the reference ones is almost perfect, as already discussed in the previous section concerning the conditional approach with default setting.

No significant improvements are instead observed for higher number of samples (orange dashed line in Fig. 15).

Fig. 16 shows the COVs of $v_{D}(d)$ and $d(v)$ for the different number of analyses and the various EDPs considered. In general, the COVs reduce passing from the case with 1 sample to the case with 30 samples per $I M$ level. However, it is not possible to identify a common pattern between the trends of the COVs for the various EDPs considered, except for the already observed tendency that the COVs of $v_{D}(d)$ increases for decreasing values of the MAF of exceedance.

With one sample per $I M$, the values of the COV for the maximum displacement demand $u$ are similar to the ones obtained with the default settings of the conditional method, but this is only for MAFs of exceedance $v_{D} \geq 10^{-3}$ such that the system response is elastic. For $v_{D}<10^{-3}$, due to the lower efficiency of the $I M$, the response dispersion notably increases and the COVs attain values up to three times higher than the reference ones. Very high COVs are also observed for the other EDPs when one single sample is used.

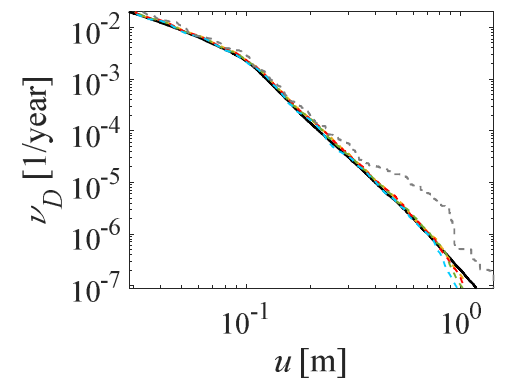

a)

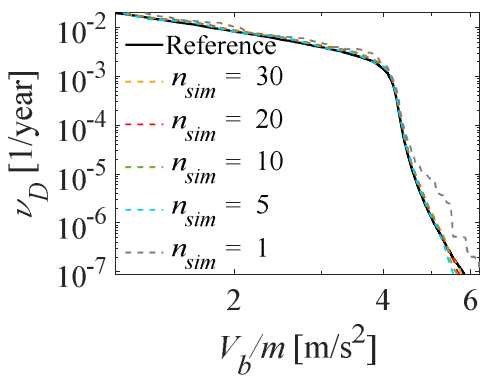

b)

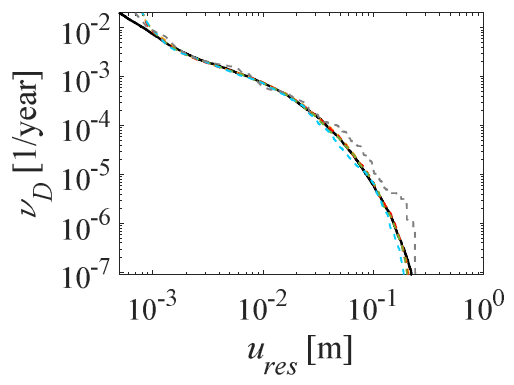

c)

Fig. 15 Influence of the number of analysis per $I M$ level on the biasedness of the average hazard curves. 


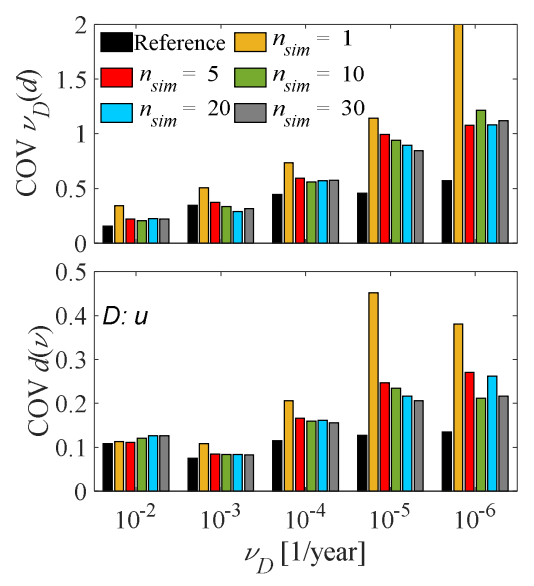

a)

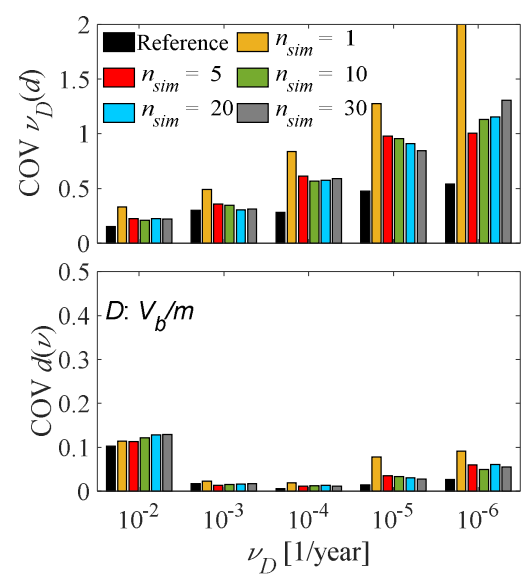

b)

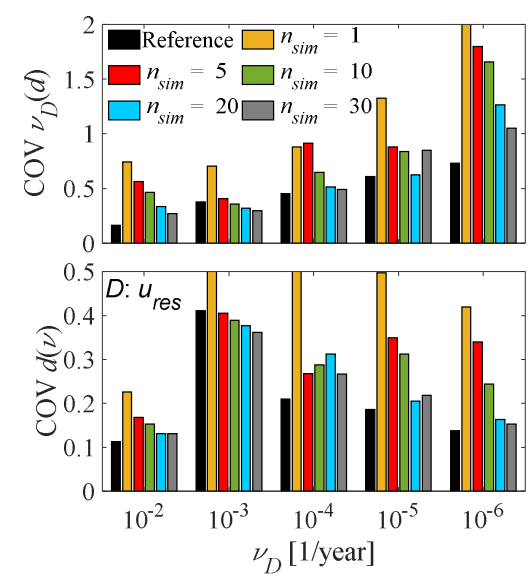

c)

Fig. 16 Effect of the number of analyses per $I M$ level on the COVs of the conditional approach and comparison with the reference values from Subset Simulation. COVs of $\boldsymbol{v}_{D}(d)$ and $d(\boldsymbol{v})$ for the demand parameters: a) $u$, b) $V_{b} / \mathrm{m}$, c) $u_{\text {res }}$

Table 6 Estimation errors $e_{D}$ at different MAFs.

\begin{tabular}{|c|c|c|c|c|c|c|c|}
\hline \multirow[b]{2}{*}{$D$} & \multirow[b]{2}{*}{ Approach } & \multicolumn{5}{|c|}{$e_{D}[\%]$} & \multirow[b]{2}{*}{ RMSE [\%] } \\
\hline & & $v_{D}=10^{-2}$ & $v_{D}=10^{-3}$ & $v_{D}=10^{-4}$ & $v_{D}=10^{-5}$ & $v_{D}=10^{-6}$ & \\
\hline \multirow{5}{*}{$u$} & $n_{\text {sim }}=1$ & -10.146 & -6.680 & -5.067 & -7.188 & -20.330 & 25.262 \\
\hline & $n_{\text {sim }}=5$ & 0.636 & 2.496 & 2.403 & 1.168 & -0.094 & 3.712 \\
\hline & $n_{\text {sim }}=10$ & 2.230 & 3.450 & 4.243 & 3.190 & 2.303 & 7.096 \\
\hline & $n_{\text {sim }}=20$ & 2.871 & 3.084 & 4.922 & 3.475 & 1.321 & 7.470 \\
\hline & $n_{\text {sim }}=30$ & 3.836 & 3.324 & 5.221 & 3.385 & 2.077 & 8.294 \\
\hline \multirow{5}{*}{$V_{b} / m$} & $n_{\text {sim }}=1$ & -8.554 & -3.086 & -0.405 & -0.894 & -3.217 & 9.695 \\
\hline & $n_{\text {sim }}=5$ & 1.783 & 0.735 & 0.326 & 0.351 & 0.416 & 2.030 \\
\hline & $n_{\text {sim }}=10$ & 3.732 & 0.821 & 0.408 & 0.775 & 1.280 & 4.124 \\
\hline & $n_{s i m}=20$ & 4.199 & 0.701 & 0.493 & 0.739 & 1.038 & 4.471 \\
\hline & $n_{\text {sim }}=30$ & 5.043 & 0.687 & 0.522 & 0.678 & 1.152 & 5.288 \\
\hline \multirow{5}{*}{$u_{\text {res }}$} & $n_{\text {sim }}=1$ & 11.257 & -25.013 & -24.551 & -22.003 & -28.991 & 51.766 \\
\hline & $n_{\text {sim }}=5$ & 16.987 & -9.644 & -9.822 & 0.989 & -8.407 & 23.446 \\
\hline & $n_{\text {sim }}=10$ & 16.658 & -2.679 & -4.726 & 3.783 & -0.427 & 17.931 \\
\hline & $n_{\text {sim }}=20$ & 11.527 & -0.632 & 1.927 & 3.910 & 2.269 & 12.546 \\
\hline & $n_{\text {sim }}=30$ & 12.912 & 0.557 & 2.804 & 3.992 & 1.185 & 13.864 \\
\hline
\end{tabular}

\subsection{Effect of the lognormality assumption for estimating $G_{D \mid I M}(d \mid i m)$}

The conditional demand model $G_{D \mid I M}(d \mid \mathrm{im})$ has been estimated so far via empirical approach (Eq. (3)).

In this subsection, the efficiency and accuracy of the "parametric approach" is investigated by considering it in combination with the interpolation strategy for $G_{D \mid I M}(\mathrm{~d} \mid \mathrm{im})$ proposed in (Bradley 2013a). In particular, the author suggests computing, via statistical inference techniques, the values of the lognormal distribution parameters (i.e., the lognormal mean and the standard deviation) of the EDP in correspondence of the $I M$ levels at which MSA is performed. Piecewise linear interpolation is then used to describe the $G_{D \mid I M}(d \mid i m)$ function for the other $I M$ values. The default setting of the conditional approach is used to perform the analyses, i.e., IM-curves with $\hat{v}=3 \cdot 10^{-7}$ discretized in $21 I M$ levels with 20 samples each.

A comparison in terms of demand hazard curves (the mean of 20 independent runs) is provided in Fig. 17, where the results of the "empirical approach" are shown by red dashed lines, and the ones from the "parametric approach" by blue dashed lines. It can be observed that the parametric approach provides curves in very close agreement with the reference ones, for all the demand parameters except for the residual displacement (Fig. 17c), for which a biased trend is shown, suggesting that the lognormal function cannot adequately represent the probabilistic distribution of this parameter. 


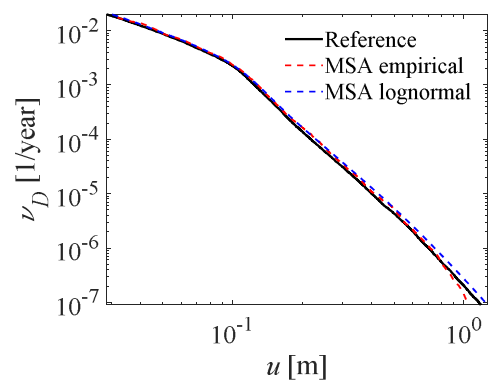

a)

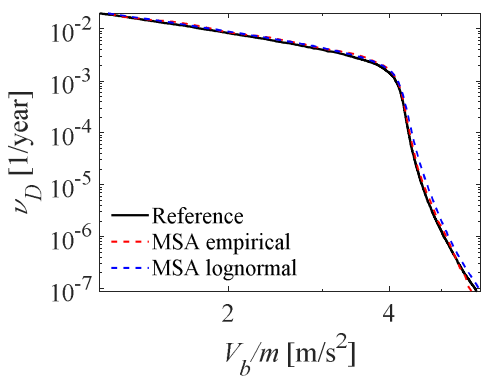

b)

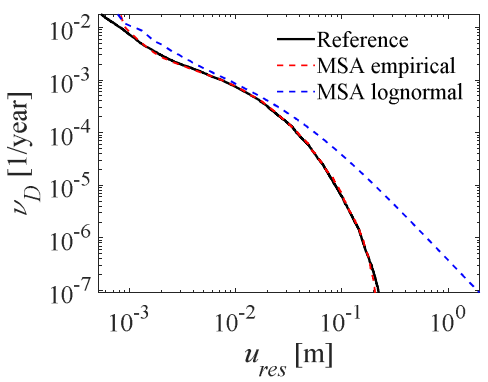

c)

Fig. 17 Effect of the lognormal assumption for computing the conditional function $G_{D \mid I M}$.

In order to investigate further this aspect, the Shapiro-Wilk test is used to assess the goodness of the lognormality assumption for the conditional demand in terms of this specific demand parameter. Such test provides the probability $\left(p_{S W}\right)$ of rejecting, at the 5\% significance level, the null hypothesis that the logarithmic values of the samples follow a normal distribution, when this is hypothesis is true. Hence the lognormality assumption is rejected whenever $p_{S W}<5 \%$. However, it is worth noting that $p_{S W}$ values higher than $5 \%$ do not rigorously prove that the response parameters follow a lognormal distribution, but only provide evidence that the null hypothesis (of the samples coming from a normally distributed population) cannot be rejected and hence the lognormality assumption might hold for the available samples (Tubaldi et al. 2015). In this sense, the $p_{S W \text {-values summarised }}$ in the following tables are used to identify the cases in which the lognormal assumption weakly fits the EDP|IM distributions. Fig. 18(a-c) show, in form of bar-plot, the percentage $p_{S W}$-values stemming from the $G_{D \mid I M}(d \mid i m)$ distributions of three independent conditional simulations (run 1, 10, 16). In the figures, the $p_{S W}$-values obtained at each of the $21 I M$ levels are illustrated. Moreover, the significance percentage threshold of 5.0\% is highlighted by a horizontal red dashed line in order to ease the identification of the cases in which the lognormality assumption is rejected, i.e., with $p_{S W}$-values $<5.0 \%$. It can be observed that there are several cases in which the assumption is rejected. Thus, the Shapiro-Wilk test confirms that the lognormal distribution is not suitable to describe the distribution of this specific demand parameter.

For sake of completeness, the lack of fit of the lognormal distribution is also displayed in Fig. 19, where the empirical (black solid line) and parametric (red dashed line) cumulative distribution functions (CDFs) $G_{D \mid I M}$ are compared for the same three simulation runs discussed above (i.e., run $1,10,16$ ). In particular, these plots refer to the CDFs computed at the $17^{\text {th }} I M$ level of MSA, corresponding to a low value of $p_{S W}$ in the Shapiro-Wilk test.

It is worth noting that the unsuitability of the lognormal fitting for the $U_{\text {res }}$ parameter observed here is in contrast with the findings of a previous study (Ruiz-García and Miranda 2006). However, it shall be noted that in (Ruiz-García and Miranda 2006) the authors used the Kolmogorov-Smirnov (KS) statistical test to evaluate the lognormality assumption. This test differs from Shapiro-Wilk test, the latter being adopted in the present study mainly because of its generally acknowledged better performance for small sample sizes (Shapiro and Wilk 1965). Nevertheless, despite it may be wise to carefully adopt the lognormal assumption for the "less common" demand parameters, the issue concerning $U_{\text {res }}$ should deserve a deeper investigation.

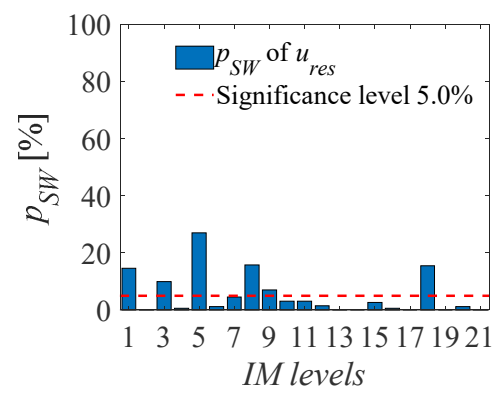

a)

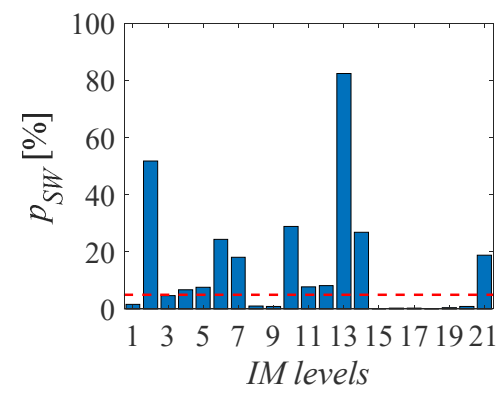

b)

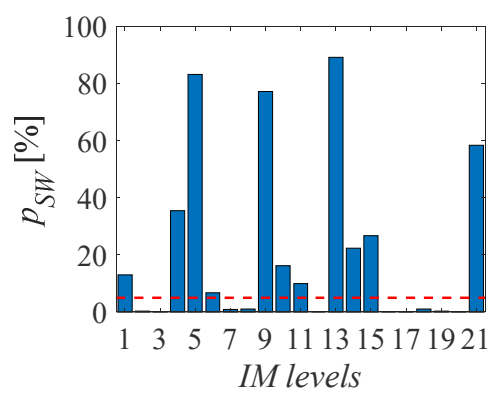

c)

Fig. 18 Shapiro-Wilk $p_{S W}$ values [\%] of the lognormality test performed on the $G_{D \mid I M}(d \mid i m)$ function of the demand parameter $u_{\text {res. }}$ Plots from three of the 20 simulation runs: a) 1, b) 10 , c) 16 . 


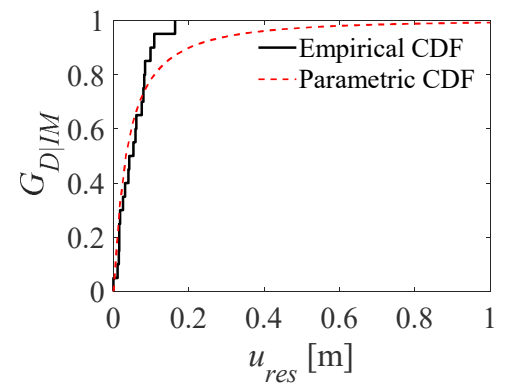

a)

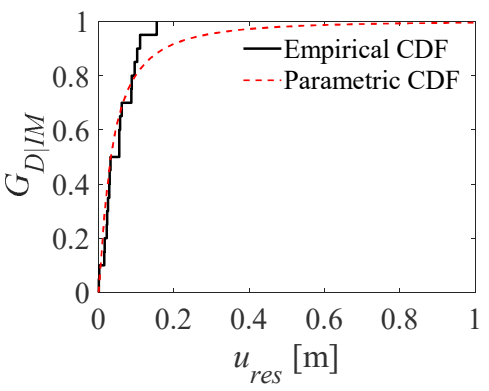

b)

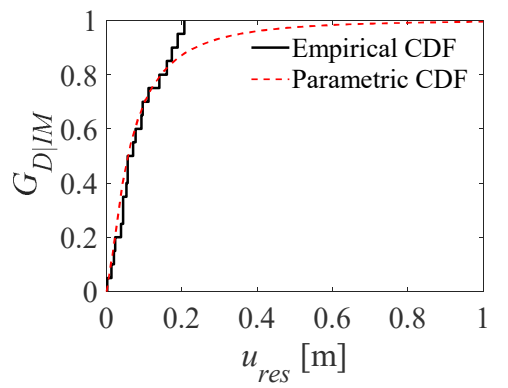

c)

Fig. 19 Empirical vs parametric $G_{D \mid I M}$ of $u_{\text {res }}$ at the $17^{\text {th }} I M$ level for three of the 20 simulations: a) 1, b) 10 , c) 16 .

Finally, the influence of the number of analyses at each $I M$ level on the demand hazard estimates obtained via the parametric evaluation of $G_{D \mid I M}$ is assessed. This investigation is similar to the one carried out in the previous Subsection 4.4, with the only difference that the conditional demand is herein estimated parametrically. Fig. 20 shows the demand hazard curves obtained by varying the number of structural analyses per $I M$ level as follows: 5, 10, 20, and 30 analysis per $I M$ level. It is observed that 5 analyses per $I M$ level might be not sufficient to reach a proper statistical characterization of the function $G_{D \mid I M}$, which introduces a source of bias on the demand hazard curves. With 10 analyses per $I M$ the estimation errors are notably reduced, and 30 analyses per $I M$ provide no remarkable improvement with respect to the case with 20 analyses per $I M$ level. Thus, a number of 10 analyses per $I M$ (for a total of 210 simulations) is sufficient to obtain a good estimation of the demand hazard. This is valid for all the EDPs, except for the already discussed residual displacements $u_{\text {res }}$.

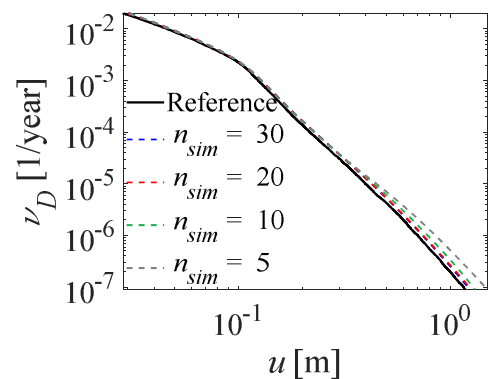

a)

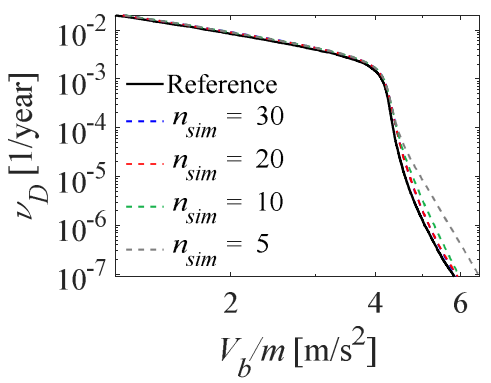

b)

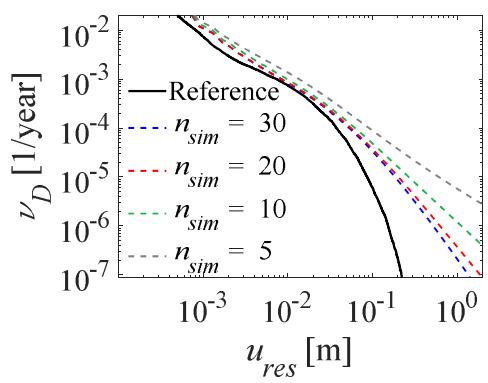

c)

Fig. 20 Influence of the number of analysis per IM-stripe on the accuracy of the lognormal assumption.

\subsection{Effect of the $I M$ hazard curve truncation}

In theory, the convolution integral in Eq. (2) should be defined over an infinite domain of possible $I M$ values, but in practice an upper bound for $I M$ should be introduced to solve numerically the problem. The effect of the lower bound is not considered herein, since the value $\bar{v}=0.3161 /$ year of the MAF of exceedance of the $I M$ is high enough that no significant earthquakes are excluded from the analysis. This subsection assesses the influence of the choices concerning the upper bound of the $I M$ hazard curve (identified by the MAF value $\hat{v}$ ) on the demand hazard estimate. For this purpose, all the controlling parameters are kept fixed to the default values of the conditional method, except for the cut-off value $\hat{v}$ of the $I M$ hazard curve and the number of $I M$ levels. For what concerns $\hat{v}$, besides the default value $\hat{v}=3 \cdot 10^{-7}$, the following ones are investigated: $1.5 \cdot 10^{-4}\left(\approx 10^{-4}\right), 9.6 \cdot 10^{-6}(\approx$ $\left.10^{-5}\right), 1.2 \cdot 10^{-6}\left(\approx 10^{-6}\right)$. The corresponding $I M$ hazard curves derive from the reference one (obtained by performing Subset Simulations with $p_{0}=0.5$ and $l=20$ ) by retaining, respectively, only the first 12,16 and $19 I M$ levels, hence there is no need to recompute the hazard curves for each of the analysed cases. As a consequence, although the total number of $I M$ levels considered is different, the same $I M$ levels and the same sets of ground motions are used (up to the truncation level) to perform MSA in all of the cases analysed. The number of dynamic analyses performed per $I M$ level is equal to 20 , as in the default case.

In Fig. 21, for each demand parameter and for each case of $I M$-curve truncation, the average of the 20 demand hazard curves obtained through the conditional approach (in coloured hatched lines) are compared to the reference average solution provided by Subset Simulation (in black solid line). Moreover, in order to quantify the level of bias of the expected value of the conditional estimator, the percentage estimation errors deriving from the conditional simulations with respect to the reference solutions provided by Subset Simulation are reported in Table 7, together with the RMSE values. It can be observed that the $I M$ truncation at $\hat{v} \approx 10^{-4}$ (grey dashed line) leads to unreliable values of the risk of exceedance below $v_{D}=10^{-3}$, with estimation errors attaining values around $60 \%$ and the RMSE values varying from $12 \%$ - to $72 \%$ depending on the EDP. The $I M$ truncation at $\hat{v} \approx 10^{-5}$ (green dashed line) instead, is found to be sufficient to achieve reliable estimates of the demand hazard up to MAFs around $v_{D}=10^{-4}$ - 
$10^{-5}$. For lower $v_{D}$ values, the errors become larger than $15 \%$, and the RMSE values vary from $6 \%$ - to $27 \%$, depending on the EDP.

Finally, the demand hazard curves obtained with an $I M$ hazard curve truncation at $\hat{v} \approx 10^{-6}$ (red dashed line) as well as those with $\hat{v}=3 \cdot 10^{-7}$ (blue dashed line) show a very good match with the reference curves, even at the lower MAF of exceedance, i.e., $v_{D}$ $<10^{-5}$. The estimation errors are always lower than 5\%, with two exceptions only, in which, however, the errors do not exceed the $12 \%$. The RMSE values, in these cases, vary from $4 \%$ - to $12 \%$, depending on the specific demand parameter. The results presented above can be explained as follows: the mean annual rate of exceedance of the largest demand thresholds $d$ is mainly influenced by earthquakes with high $I M$ levels, hence truncating the $I M$ hazard curve at too low $I M$ levels implies neglecting such contribution and this leads to the underestimation of the seismic risk. To support this explanation, and also to verify the limits of the numerical integration, a disaggregation of the seismic demand hazard (Baker et al. 2005) is carried out. Fig. 22 shows the disaggregation of the hazard curve of the maximum displacement with respect to the $I M$ levels. More precisely, four different threshold values of the demand parameter are selected, corresponding to the following values of the MAF of exceedance $v_{D}$ (evaluated on the reference mean conditional solution): $10^{-3}, 10^{-4}, 10^{-5}, 10^{-6} 1$ year. For each threshold $d(v)$ (with the abovesaid $\left.v_{D}\right)$, the $I M$ levels contributing to the probability of exceedance $\mathrm{P}[D>d(v)]$ are displayed in form of histogram, with one plot for each $v_{D}$. According to the disaggregation presented in Fig. 22, the $I M$ hazard curve with $12 I M$ levels (i.e., truncated at $\hat{v} \approx$ $10^{-4}$, grey dashed line in Fig. 21a) can be used to properly characterize the demand hazard up to $v_{D}=10^{-3}$, because the $I M$ levels mainly contributing to this part of the demand hazard curve are from 13 below. The $I M$ levels from 13 to 18 (Fig. 22a) provide a negligible contribution. According to the disaggregation results given in Fig. 22b, the major contribution to the MAF of exceedance $v_{D}=10^{-4}$ comes from the $I M$ levels from 12 to 16 , and this explains why the $I M$ hazard curve with $16 I M$ levels (i.e., truncated at $\hat{v} \approx 10^{-5}$, green dashed line in Fig. 21a) is able to characterize well the demand hazard up to $v_{D}=10^{-4}$. According to the disaggregation of Fig. 22c, the major contribution to the risk of exceedance of $d(v)$ with $v_{D}=10^{-5}$ comes from the $I M$ levels from 15 to 19 , and this explains why the $I M$ hazard curve with $19 I M$ levels (i.e., truncated at $\hat{v} \approx 10^{-6}$, red dashed line in Fig. $21 \mathrm{a}$ ) is able to estimate with accuracy the demand hazard up to $v_{D}=10^{-5}$. On the other hand, the same $I M$ hazard curve yields a slight underestimation around $v_{D}=10^{-6}$ and below, and this can be explained by looking at Fig. 22d, where it can be observed how the contribution to the risk provided by the disregarded $I M$ levels 20 and 21 is actually non-negligible.

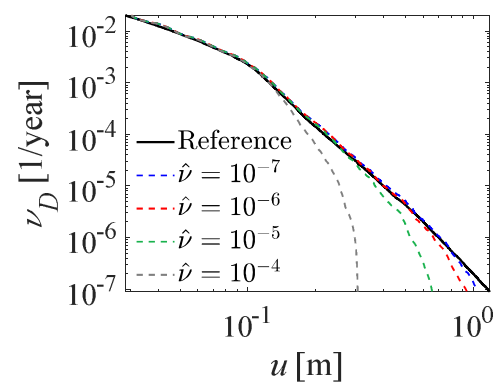

a)

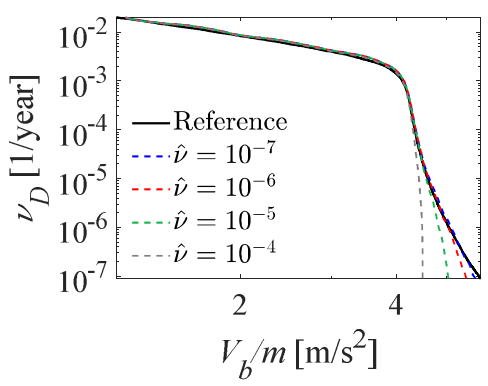

b)

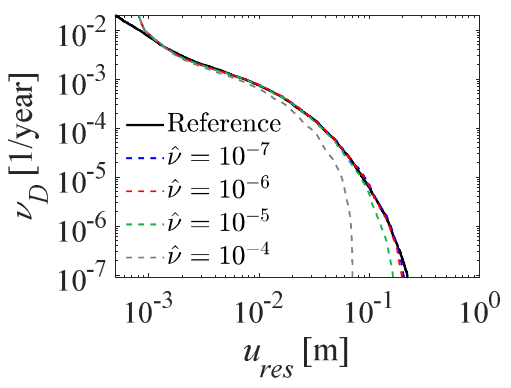

c)

Fig. 21 Influence of the $I M$ hazard curves truncation on the biasedness of the demand hazard curves.

Table 7 Estimation errors $e_{D}$ at different MAFs.

\begin{tabular}{l|l|l|l|l|l|l|l}
\hline & & \multicolumn{5}{|c|}{$e_{D}[\%]$} & \\
\cline { 3 - 8 }$D$ & Approach & $v_{D}=10^{-2}$ & $v_{D}=10^{-3}$ & $v_{D}=10^{-4}$ & $v_{D}=10^{-5}$ & $v_{D}=10^{-6}$ & RMSE [\%] \\
\hline \multirow{4}{*}{$u$} & $\hat{v} \approx 10^{-4}$ & 2.302 & -0.389 & -15.561 & -35.995 & -57.653 & 69.765 \\
& $\hat{v} \approx 10^{-5}$ & 2.842 & 2.810 & 2.598 & -8.040 & -25.470 & 27.131 \\
& $\hat{v} \approx 10^{-6}$ & 2.869 & 3.047 & 4.381 & 0.047 & -5.322 & 8.064 \\
& $\hat{v} \approx 10^{-7}$ & 2.871 & 3.084 & 4.922 & 3.475 & 1.321 & 7.470 \\
\hline \multirow{5}{*}{$V_{b} / m$} & $\hat{v} \approx 10^{-4}$ & 3.556 & -0.018 & -0.814 & -3.860 & -11.165 & 12.364 \\
& $\hat{v} \approx 10^{-5}$ & 4.166 & 0.620 & 0.314 & -0.695 & -4.375 & 6.121 \\
& $\hat{v} \approx 10^{-6}$ & 4.198 & 0.692 & 0.474 & 0.473 & -0.144 & 4.309 \\
& $\hat{v} \approx 10^{-7}$ & 4.199 & 0.701 & 0.493 & 0.739 & 1.038 & 4.471 \\
\hline \multirow{4}{*}{$u_{\text {res }}$} & $\hat{v} \approx 10^{-4}$ & 10.968 & -15.576 & -23.190 & -35.428 & -55.480 & 72.345 \\
& $\hat{v} \approx 10^{-5}$ & 11.495 & -2.044 & -1.449 & -4.415 & -16.708 & 20.906 \\
& $\hat{v} \approx 10^{-6}$ & 11.525 & -0.646 & 1.726 & 2.578 & -0.553 & 11.966 \\
& $\hat{v} \approx 10^{-7}$ & 11.527 & -0.632 & 1.927 & 3.910 & 2.269 & 12.546 \\
\hline
\end{tabular}




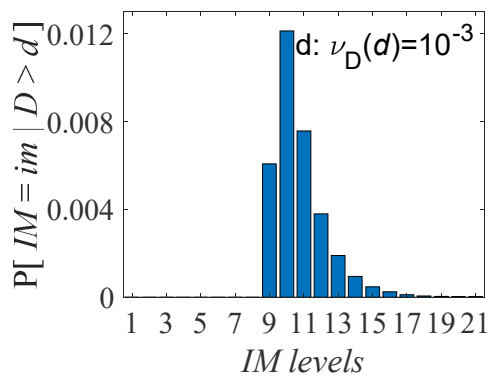

a)

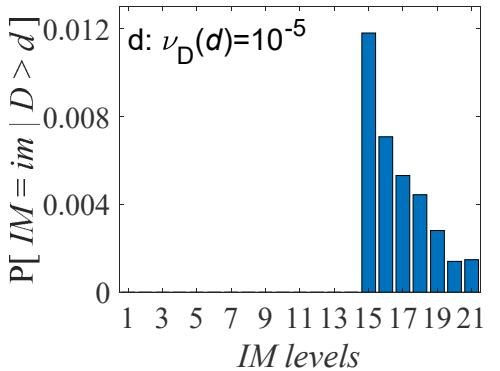

c)

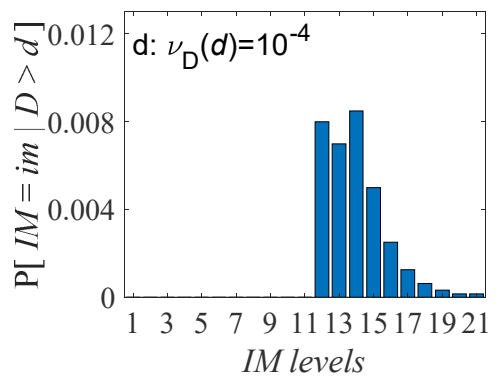

b)

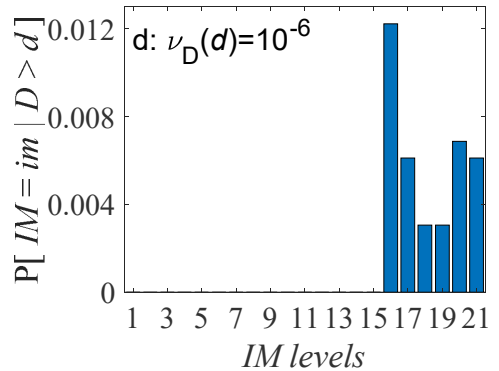

d)

Fig. 22 IM levels contributing to exceedance of different threshold values $d$ corresponding to fixed $u$ values $\left(10^{-3}, 10^{-4}, 10^{-5}, 10^{-6} 1 /\right.$ year $)$. Demand parameter: maximum displacement $u$.

\section{Method validation: analysis of a MDOF steel building}

This last section investigates the bias and accuracy of the conditional method obtained for the MDOF model of the steel building. The dynamic behaviour of this model is more complex and realistic than that of the SDOF model, also due to the contribution of higher modes that might affect the seismic response. In Subsection 5.1, the reference solution obtained via Subset Simulation is discussed, whereas Subsection 5.2 provides details about the reference solution from the conditional method. Finally, in Subsection 5.3 the effect of the controlling parameters of the conditional approach is analysed.

The five following demand parameters are considered to monitor the performance of the system and evaluate the accuracy of MSA: the maximum interstory drift ratio $I D R$, directly related to the damage level on both structural and non-structural (displacement-sensitive components, partition walls, etc.) elements; the maximum absolute floor acceleration $A$, providing indirect information on the response of acceleration-sensitive non-structural components; the maximum absolute base-shear $V_{b}$, as an indicator of the global rate of work of the whole structural system as well as the foundations; the top-floor displacement $u_{\text {top }}$, as indicator of the global system deformability; the maximum residual interstory drift ratio $I D R_{\text {res }}$, providing insights into post-earthquake retrofit costs and activities.

\subsection{Reference solution via Subset Simulation for the MDOF system}

The reference solutions used to assess the efficiency of the conditional probabilistic method are provided from a direct simulation approach via Subset Simulation. A reliable estimate of the risk up to very low MAF of exceedances (in the range $10^{-5}-10^{-6}$ ) is desirable and thus the same setting adopted for the SDOF system is used here: $p_{0}=10 \%, l=7$, and $n_{\operatorname{sim}}=500$, for a total amount of analyses per simulation equal to 3500. A set of 20 independent replicates of Subset Simulation is performed and then the averaged demand hazard curves are taken for each demand parameter introduced before. Unlike the case of SDOF system analysed before, performing nonlinear dynamic analyses $(3500 \times 20=70000)$ with the MDOF model has a quite high computational cost.

The demand hazard curves for the monitored demand parameters are plotted in Fig. 23. The results obtained for independent runs of Subset Simulation are shown by grey dotted lines, and the corresponding average demand hazard curves are plotted with black solid lines. The various EDPs are characterized by different trends of the demand hazard curves, as expected. For instance, the maximum base shear (Fig. 23b) exhibits a visible change of slope due to the yielding of the structural components, which occurs gradually and this produces a smoother transition from the elastic to the plastic phase compared to the SDOF system. In the hazard curves of the residual interstory drift (Fig. 23d), small demand values are shown (drift of the order of magnitude of $0.1 \%$ ) for the hazard level corresponding to $v_{D}>0.0021 /$ year. The presence of non-null residual drift (though very small) for 
$v_{D} \approx 10^{-2} 1$ year follows from the elastoplastic constitutive law with smooth elastic-to-plastic transition used within the fiber sections of the finite element model.

The confidence of the estimates is quantified by the COVs of $v_{D}(d)$ and $d(v)$, shown in Fig. 24. The COVs of $v_{D}(d)$ span from 0.2 to 1.5 , with higher values corresponding to lower MAF of exceedances. The COVs of $d(v)$ are always lower than 0.4 , and their trend of variation with the MAF is irregular and strongly depends on the specific demand parameter $D$. The comments given for the SDOF system still hold for the current case study. However, a slightly higher dispersions can be generally observed for the current model, with values of COVs of $v_{D}(d)$ that can be above 1.0 for the case of the absolute accelerations and base shear. This increase of COVs with respect to the SDOF model is related to the effect of the higher modes, that affect more significantly these response quantities compared to the kinematic ones.

Both the mean hazard curves and the COVs given here are assumed as reference solutions in the next subsection to test the results from the conditional approach.

In order provide the reader with some practical information about the expected structural performance, the values of the demand parameter thresholds denoting the attainment of the main limit states are recalled below. For instance, collapse limit state for new steel buildings (FEMA-350 2000a) corresponds to $I D R \geq 0.1$, which in this application corresponds to annual rates of exceedance roughly equal to $10^{-6}$ (Fig. 23a). For what concerns the maximum residual drift demand (FEMA-350 2000b) (RuizGarcía and Miranda 2006), instead, this should not exceed the limit 0.01 for Life Safety and 0.05 for Collapse Prevention performance conditions; in this application such threshold values correspond to annual rates of exceedance roughly equal to $10^{-}$ ${ }^{4}$ and $10^{-5}$, respectively (Fig. 23e).

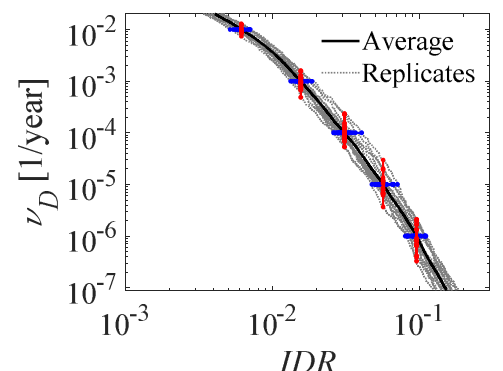

a)

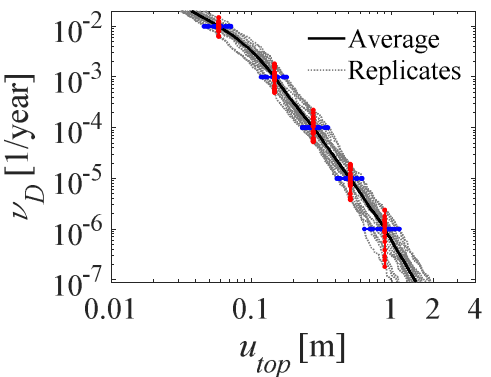

d)

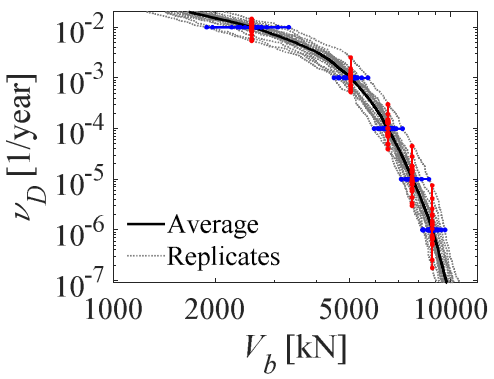

b)

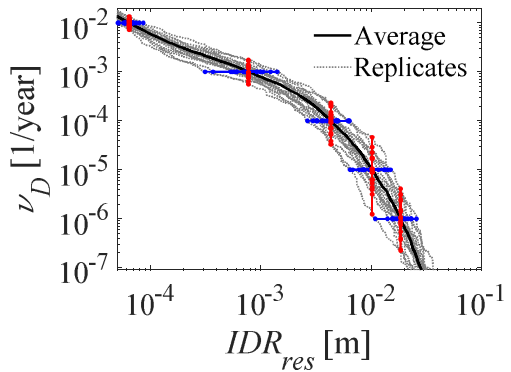

e)

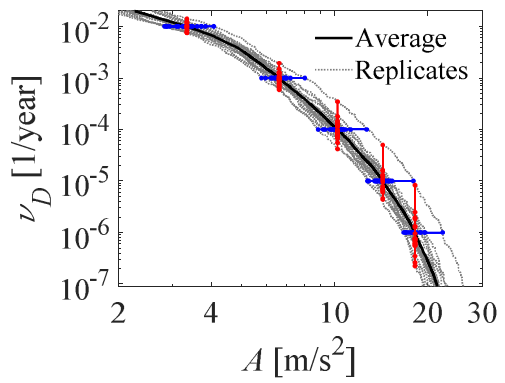

c)

Fig. 23 Reference demand hazard curves obtained averaging 20 independent runs of Subset Simulations.

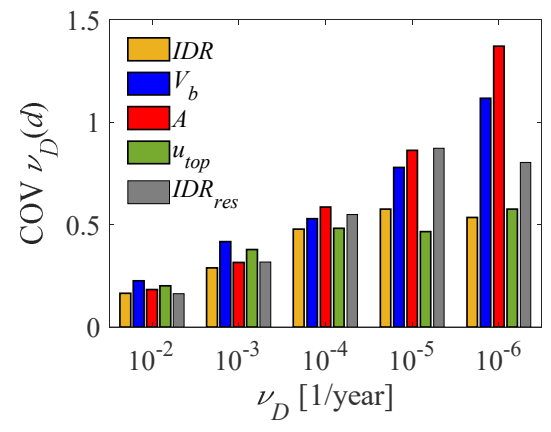

a)

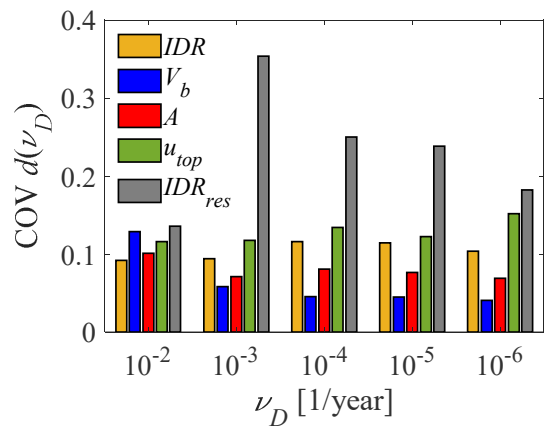

b)

Fig. 24 Reference COVs of a) $v_{D}(d)$ and b) $d(v)$ from Subset Simulation for different EDPs. 


\subsection{Reference solution via conditional approach for the MDOF system}

The reference conditional solution provided by the parametric study carried out in previous Section 4 consists of MSA performed at $21 I M$ levels with 20 analyses each, with an $I M$ hazard curve properly truncated at $\hat{v} \approx 10^{-7}$. This setting is now used to validate the suitability of the method in dealing with the more complex structural system introduced above. In particular, in this Subsection the reference conditional solution for the MDOF model is discussed. In the following subsection, the influence of the main sets of controlling parameters on the demand hazard estimates for the MDOF system is assessed. In particular, the following settings and setup choices are analysed: $I M$ curve discretisation, the number of analyses per $I M$ level, and the use of empirical or parametric approach to estimate $G_{D \mid I M}$.

Fig. 25 shows the demand hazard curves for all of the EDPs relevant to the performance of the MDOF system. The mean reference solution from the conditional approach (red dashed line) is compared with the mean reference solution provided by Subset Simulation (black solid line). The curves represent the average of 20 independent simulations. For sake of completeness, the demand hazard curves obtained with single runs of MSA are also plotted in the figure with grey dotted lines.

First of all, in Fig. 25 it is possible to observe a satisfactory match between the conditional and the reference curves, which again proves the unbiasedness, on average, of the MSA estimator with the reference setting. This is also confirmed by the small estimation errors $e_{D}$ observed for all of the monitored demand parameters, collected in Table 8. Moreover, it is worth noting that the order of magnitude of the error values observed in this Section for the MDOF system (Table 8) is the same as for the SDOF system analysed before (Table 4).

The COVs are displayed in Fig. 26. As noted for the estimation errors, a substantial similarity is also observed between the values observed for the MDOF system (Fig. 26) and those previously observed for the SDOF system (Fig. 11). As a general trend, the COVs observed for the conditional method are slightly higher than the reference ones from Subset Simulation. On the contrary and quite interestingly, the COVs of the absolute acceleration provided by the conditional approach are always comparable or even lower than those obtained via Subset Simulation.

To conclude this part of the study and in light of the outcomes discussed so far, the statistical precision of the conditional method remains essentially unchanged by changing the system analysed, and the increase of complexity and in the degrees of freedom do not affect the properties of the estimator. This confirms the conditional method as suitable tool able to provide demand hazard estimates that are on average unbiased.

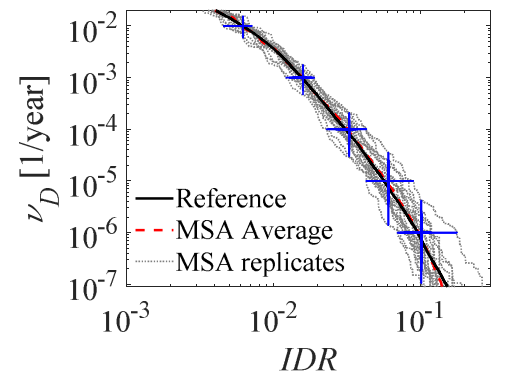

a)

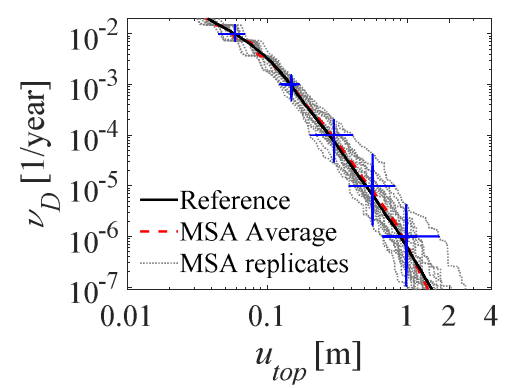

d)

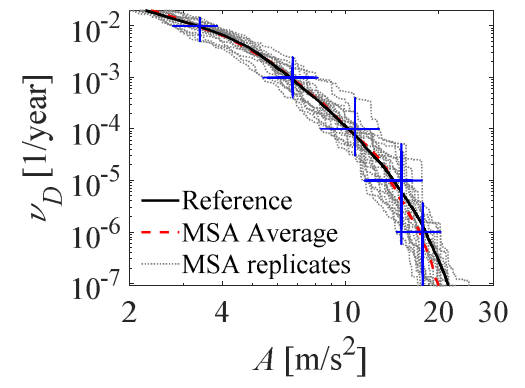

b)

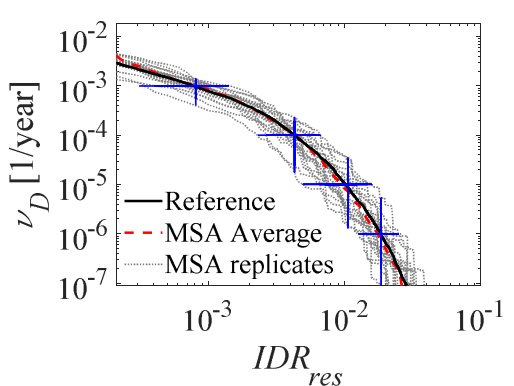

e)

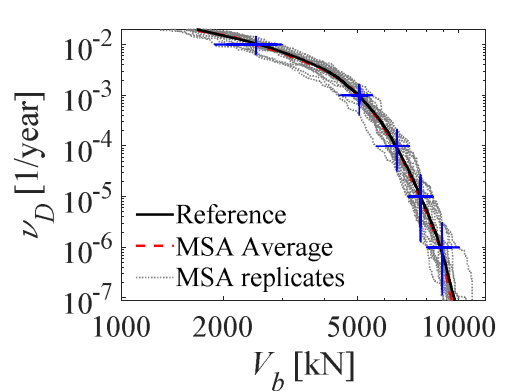

c)

Fig. 25 Conditional simulation replicates and corresponding average curves compared to the reference solution provided by Subset Simulations, for different demand parameter. Cross marks highlight the cases at which the COVs are evaluated. 


\begin{tabular}{l|lllll|l}
\hline \multirow{2}{*}{$D$} & \multicolumn{5}{|c|}{$e_{D}[\%]$} & \multirow{2}{*}{ RMSE [\%] } \\
\cline { 2 - 6 } & $v_{D}=10^{-2}$ & $v_{D}=10^{-3}$ & $v_{D}=10^{-4}$ & $v_{D}=10^{-5}$ & $v_{D}=10^{-6}$ & \\
\hline$I D R$ & -2.723 & 0.887 & 3.193 & 3.442 & 2.070 & 5.876 \\
$A$ & -4.230 & -0.467 & -0.224 & -0.490 & -0.172 & 4.293 \\
$V_{b}$ & -0.980 & -0.281 & -0.240 & -2.735 & -4.636 & 5.484 \\
$u_{\text {top }}$ & -3.646 & 0.992 & 4.084 & 4.277 & 3.578 & 7.877 \\
$I D R_{\text {res }}$ & 6.732 & 7.681 & 0.124 & -6.922 & -4.774 & 11.389 \\
\hline
\end{tabular}

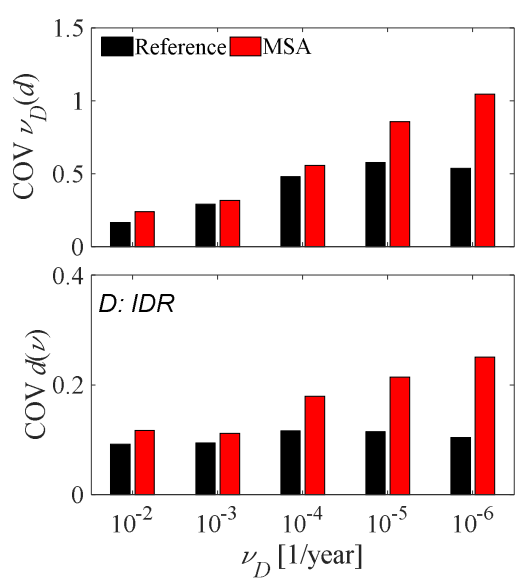

a)
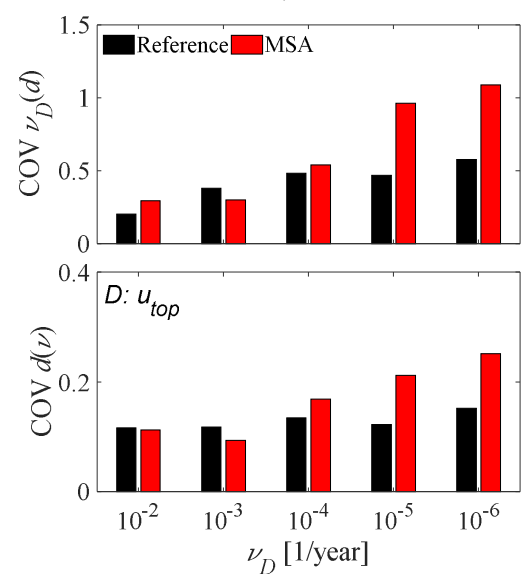

d)

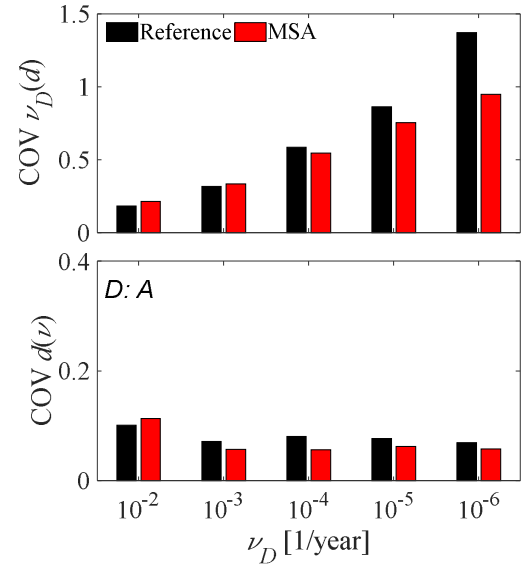

b)

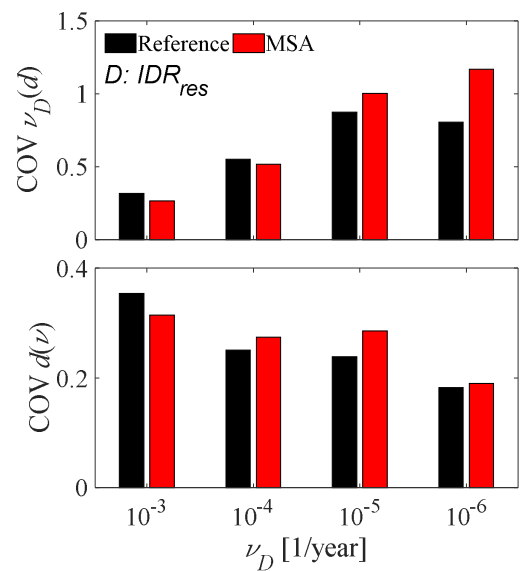

e)

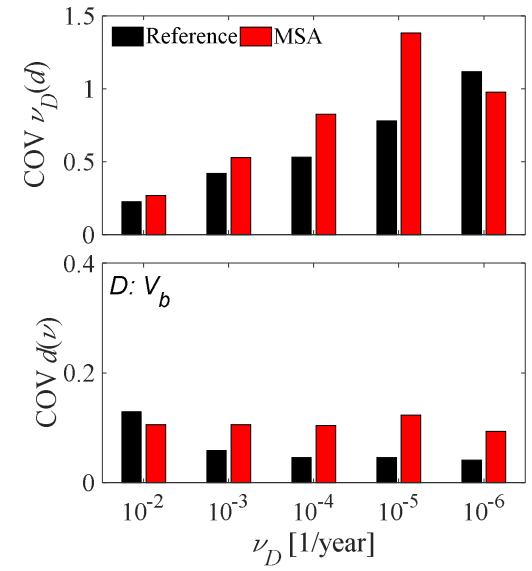

c)
698

Fig. 26 Comparison of the COVs of the conditional solution with the reference values from Subset Simulation. COVs of $v_{D}(d)$ and $d(v)$ for different demand parameters: a) $I D R$, b) $A$, c) $V_{b}$, d) $u_{t o p}$, e) $I D R_{\text {res. }}$

\subsection{Effect of the controlling parameters of the conditional approach}

The influence of the most critical parameters controlling the conditional approach applied to the MDOF system is now assessed. In particular, based on the outcomes provided by the previous parametric study (Section 4), the following combinations of the most relevant controlling parameter are analysed and compared:

- MSA performed on $21 \mathrm{IMs}$ and one single ground motion sample per IM level, for a total of 21 analyses (effect of the number of analyses per $I M$ level);

- $\quad$ MSA performed on 6 IMs and 20 ground motion samples per $I M$ level, for a total of 120 analyses (effect of the $I M$ curve discretisation);

- $\quad$ MSA performed on 21 IMs and 20 ground motion samples per IM level (for a total of 420 analyses) with demand model $\boldsymbol{G}_{\boldsymbol{D} \mid \boldsymbol{I M}}$ build via "parametric approach" (effect of the lognormal assumption on the D|IM distribution).

In all cases, the parameter $\hat{v}$ (IM hazard curve truncation) is kept fixed at the reference value $\hat{v}=3 \cdot 10^{-7}$.

Fig. 27 shows the mean demand hazard curves corresponding to the settings listed above, together with the reference curves obtained via Subset Simulation. All the curves refer to the average of 20 independent simulations. 
For what concerns the effect of the number of analyses per $I M$ level, the mean demand hazard curves related to the case with one single ground motion sample (red dot-dashed lines) do not deviate significantly from the reference solution, although a certain level of bias can be detected, in particular at the lower rates of exceedance and for the demand parameter absolute acceleration (A). Such limited influence of this controlling parameter is mainly related to efficiency of the adopted IM and the use of hazardconsistent ground motion samples that are different at each intensity level, thus overcoming potential issues of sufficiency of the adopted $I M$. On the other hand, a coarser discretisation of the $I M$ hazard curve (blue dotted lines) induces a quite significant bias, corresponding to a significant overestimation of the demand hazard curves of all the EDPs. This is consistent with the results observed for the SDOF system, where the estimation errors have always positive sign.

With regard to the effect of the lognormal assumption on the estimation of the demand model $G_{D \mid I M}$, the parametric estimate (green dashed lines) has a fine match with the reference solutions in terms of all the EDPs, except for the residual drift (Fig. 27d), in analogy to what was noted in Subsection 4.5, about the residual displacement parameter of the SDOF system. In this case, indeed, a significant deviation from the target curve is observed from MAF values of $v_{D} \approx 10^{-4}$ and below.

For what concerns the COVs (Fig. 28), there are no patterns worth to be highlighted and the trends are those already discussed in the previous subsection concerning the reference conditional solution. However, some results deserve to be discussed. In particular, the COVs corresponding to the case with one analysis per IM level (red bars in Fig. 28) are almost always the highest, implying that the adopted $I M$ is not efficient enough to compensate the lack of a large set of ground motion samples used to reproduce record-to-record variability effects. The COVs of $v_{D}$ provided with the hazard curve discretised in 6 IM levels (blue bars in Fig. 28) are also slightly over the average, in particular for the demand parameters absolute acceleration and residual drift. The latter demand parameter also shows high COVs of $d(v)$, consistently with other studies on the topic (Ruiz-García and Miranda 2006). The COVs from MSA with the lognormal assumption (green bars in Fig. 28) are always comparable to the reference conditional solution shown previously in Fig. 26.

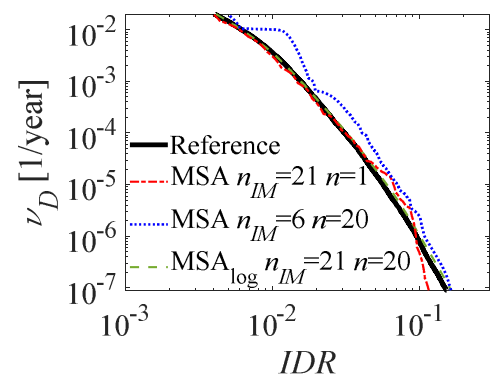

a)

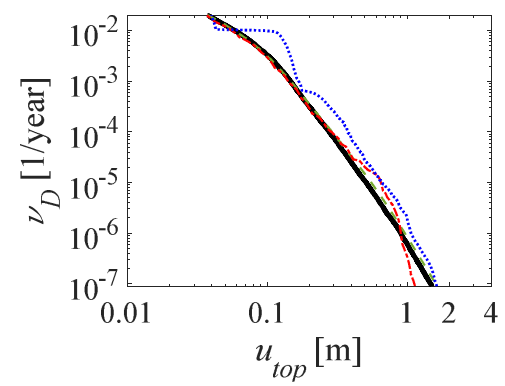

d)

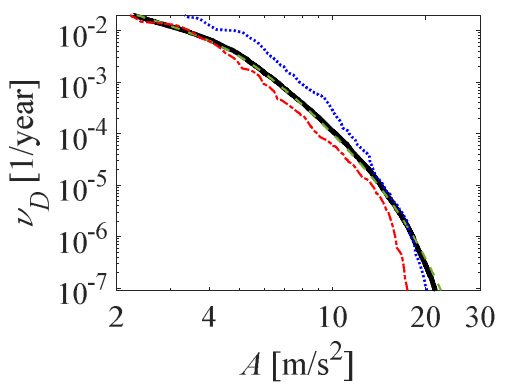

b)

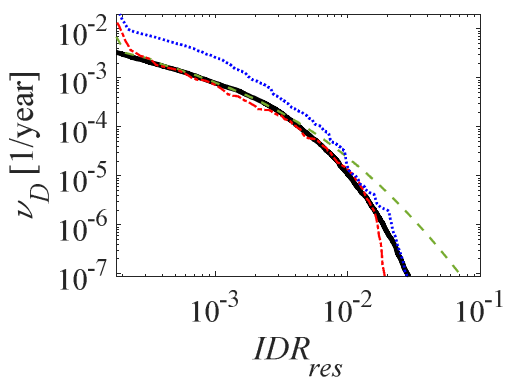

e)

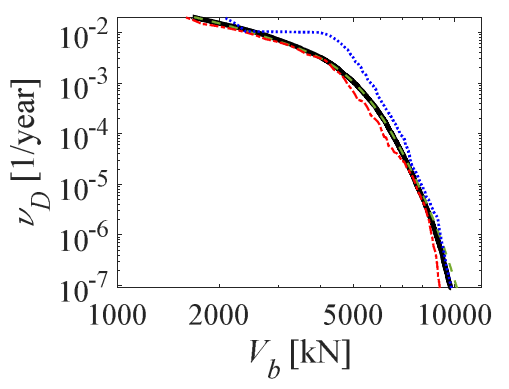

c)

Fig. 27 Conditional simulation replicates and corresponding average curves compared to the reference solution provided by Subset Simulations, for different demand parameter. 


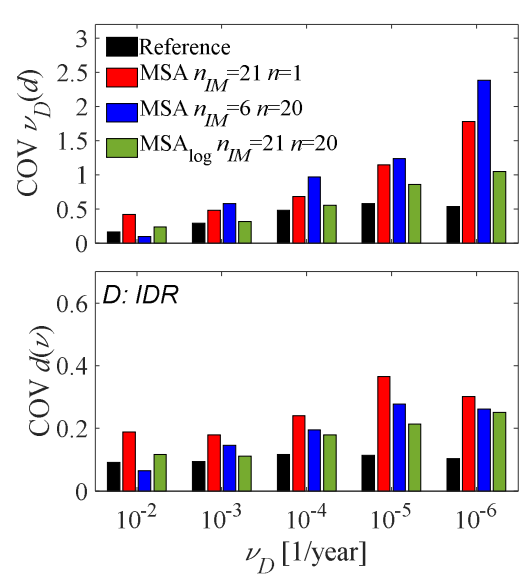

a)
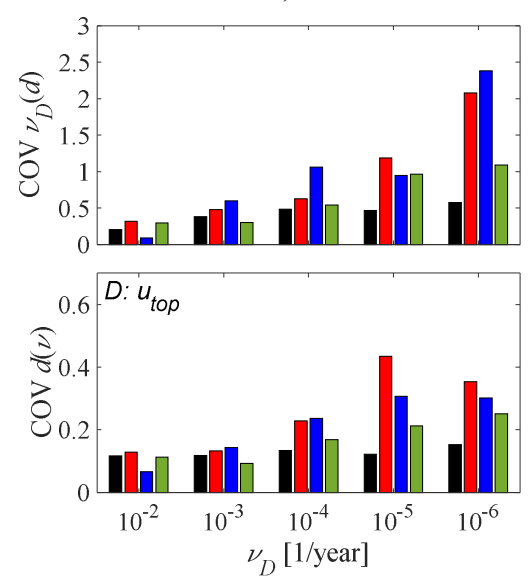

d)
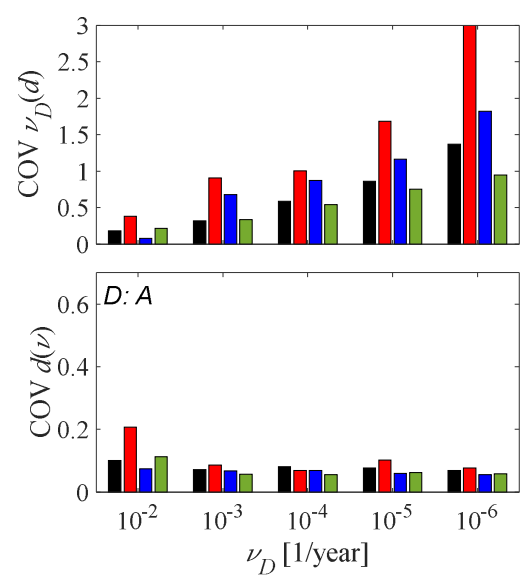

b)
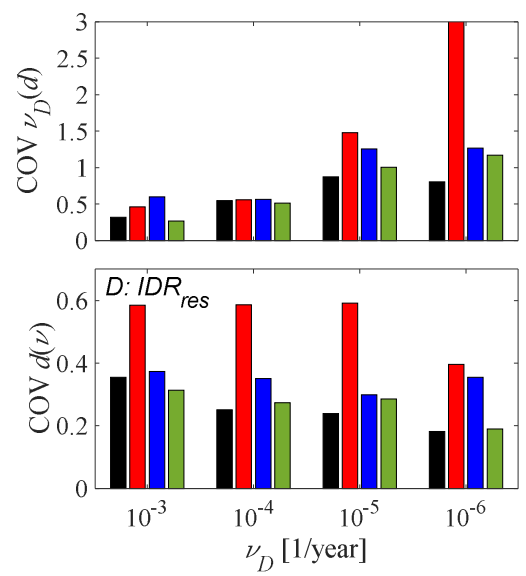

e)

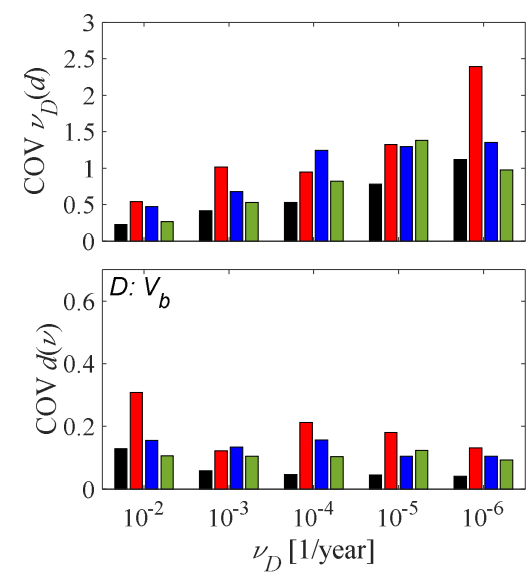

c)

Fig. 28 Comparison of the COVs of the conditional solution with the reference values from Subset Simulation. COVs of $v_{D}(d)$ and $d(v)$ for different demand parameters: a) $I D R$, b) $A$, c) $V_{b}$, d) $u_{\text {top }}$, e) $I D R_{\text {res. }}$

\section{Conclusions}

An extensive investigation on the effectiveness of conditional approaches for demand hazard evaluation has been carried out by analysing a nonlinear single-degree-of-freedom system and a multi-degree-of-freedom model of a steel building. A conditional approach based on Multiple-Stripe Analysis (MSA) has been employed, in conjunction with the spectral acceleration as intensity measure $(I M)$. Subset Simulation has been used for estimating the seismic hazard at the site and identifying, with a stochastic ground motion model, the set of records to be used for MSA at the different $I M$ levels. The demand hazard estimates obtained with the conditional approach have been compared to the ones obtained using Subset Simulation.

It has been shown that, overall, the conditional approach is quite accurate and computationally efficient, since it is able to provide demand hazard estimates that are on average unbiased, with a statistical precision only slightly lower than that of Subset simulation. To show this, a reference conditional solution has been considered, with MSA performed at 21 IM levels with 20 analyses each, and the $I M$ hazard curve truncated at the mean annual frequency of exceedance of $\hat{v} \approx 10^{-7} 1 /$ year. This setting provides a trade-off between accuracy and computational cost in performing probabilistic analyses on both the simple nonlinear SDOF and the more complex MDOF system. Indeed, the gain in terms of computational cost is noticeable, since the time required to perform a single run of the direct approach with Subset Simulation ( 7 simulation levels with 500 analyses each and a probability of exceedance governing the level-to-level transition equal to $p_{0}=10 \%$, thus corresponding to 3200 analyses) is about 8 times higher than that required by the conditional approach (with a total amount of 420 analyses).

The results of the study performed in this paper provide useful information about the influence of the various parameters controlling the quality of the solution achieved via the conditional approach, and their optimal choice.

In particular, the following main conclusions can be drawn for the problem considered:

- $\quad$ The number of $I M$ levels used to perform MSA strongly affects the accuracy of the numerical integration. In general, it is observed that the degree of overestimation on the seismic demand hazard increases using coarser discretisation of the $I M$ hazard curve. On the basis of the numerical results, a number of $I M$ levels higher than 10 seem to be sufficient to avoid accuracy issues related to the $I M$ discretisation. 
- The number of analyses performed at each $I M$ level influences the unbiasedness of the estimator, and at least 10 simulations (20 are recommended) should be carried out to properly characterise the record-to-record variability effects at a given $I M$ level. MSA performed with a single sample per IM level could be sufficient in case of very efficient IMs, however, some degree of bias cannot be avoided, particularly at the lowest MAF values (i.e., lower than $10^{-4} 1 /$ year).

- The lognormal assumption generally provides accurate results and can be used for the parametric estimation of the demand hazard of almost all the monitored demand parameters. The only exception is when the residual drifts are monitored. This is mainly due to the fact that conditional demand cannot be approximated by a lognormal distribution, particularly at the lowest MAF values (lower than $v_{\mathrm{D}} \approx 10^{-4}$ ). Thus, particular care should be taken in using the widely employed lognormal assumption to model the conditional distribution of unusual demand parameters.

- The choice of the upper bound for the $I M$ hazard curve truncation affects the accuracy of the numerical integration, and a sufficiently large upper bound of the $I M$ should be considered in order to not neglect important contributions provided by rare ground motions. In particular, an accurate characterisation of the demand hazard curves up to small failure annual rates $\left(v_{\mathrm{D}} \approx 10^{-6}\right)$ can be achieved by considering IM levels corresponding to MAF of exceedances up to $\hat{\boldsymbol{v}} \approx 10^{-7}$.

\section{Open problems and future developments}

Although extensive, the presented study does not cover all possible aspects concerning the subject at hand. A summary of the main open problems and limits of this work is provided below, setting the basis for future developments.

- Stochastic ground motion model. The adopted stochastic model is chosen for its capability to describe record-to-record variability effects. However, the variability observed in real earthquakes is not easily reproducible, and the use of the stochastic model inevitably introduces some sources of approximation. However, it is worth recalling that, for the purpose of this study the availability of a stochastic model was essential to ensure the existence of a reference solution against which to compare the results of the $I M$-based conditional approach. Future works could employ alternative stochastic ground motion models available in the literature (Rezaeian and Kiureghian 2010) (Yamamoto and Baker 2013), as well as compare the seismic risk estimates provided by natural and synthetic ground motion samples.

- Case study. The reference case study used in this paper can be considered as representative of a wide class of buildings; however, the analysis outcomes might not hold for different structural systems and it might be thus interesting to extend the study to a wider set of common building types (e.g., reinforced-concrete or masonry structures). Furthermore, the analysis of more complex structural systems might help to further assess the influence of the higher vibration modes on the performance of the conditional probabilistic method. On the other hand, more refined modelling approaches accounting for both strength and stiffness cyclic deterioration on structural elements might be considered to better evaluate the efficiency of conditional methods at the collapse condition.

- Intensity measure. The same methodology could be employed in future analyses considering other, more efficient, IMs recently proposed in the literature, such as the average spectral acceleration (Eads et al. 2015) and the filtered incremental velocity (Dávalos and Miranda 2019a).

- Demand hazard assessment. The approach followed in this study could be employed to evaluate the efficiency, accuracy and precision of other analysis methods widely employed in PBEE, such as IDA and cloud analysis, or of advanced simulation tools in alternative to Subset Simulation.

\section{Appendix}

The present Appendix provides some details about the construction of the $I M$ hazard curves $v_{I M}(\mathrm{im})$ via Subset Simulation (Au and Beck 2003). For this aim, Subset Simulation is performed by considering $l=20$ levels, each having a target intermediate exceedance probability $p_{0}=0.5$ and $n_{\text {sim }}=500$ analyses per level. Consequently, 500 ground motion samples are generated, from a stochastic ground motion model, within each of the $l$ simulation levels, which also correspond to the intervals of discretization of the $I M$ hazard curve obtained in output. Indeed, the hazard curve discretisation follows from the $I M$ intermediate thresholds generated during the Subset Simulation run.

To be precise, Subset Simulation provides $I M$ hazard curves with inferior limit corresponding to the annual rate of exceedance $\bar{v}=0.3161 /$ year, identifying the rate of occurrence of earthquakes of any magnitude between $m_{0}$ and $m_{\max }$; the superior limit, 
$\hat{v}=3 \cdot 10^{-7} 1$ year, corresponds to $\bar{v} \cdot p_{0}^{l}$. In conclusion, by including the lower bound $\bar{v}, \mathrm{v}_{I M}$ is discretized in a total of 21 points, corresponding to $n_{I M}=21 I M$ levels or stripes.

Among the 500 ground motion samples generated at each $I M$ level, a subset of $n_{\text {sim }}=20$ samples is selected to represent the recordto-record variability effects conditional on the $I M$ level.

\section{References}

Aslani H, Miranda E (2005) Fragility assessment of slab-column connections in existing non-ductile reinforced concrete buildings. Journal of Earthquake Engineering 9:777-804. doi: 10.1080/13632460509350566

Atkinson GM, Silva W (2000) Stochastic modeling of California ground motions. Bulletin of the Seismological Society of America 90:255-274. doi: 10.1785/0119990064

Au SK, Beck JL (2003) Subset Simulation and its Application to Seismic Risk Based on Dynamic Analysis. Journal of Engineering Mechanics 129:901-917. doi: 10.1061/(ASCE)0733-9399(2003)129:8(901)

Au SK, Patelli E (2016) Rare event simulation in finite-infinite dimensional space. Reliability Engineering and System Safety 148:67-77. doi: 10.1016/j.ress.2015.11.012

Au SK, Wang Y (2014) Engineering Risk Assessment with Subset Simulation

Baker JW, Cornell CA (2006) Spectral shape, epsilon and record selection. Earthquake Engineering and Structural Dynamics 35:1077-1095. doi: 10.1002/eqe.571

Baker JW, Cornell CA, Tothong P (2005) Disaggregation of seismic drift hazard. In: 9th International Conference on Structural Safety and Reliability (ICOSSAR05)

Barroso LR, Winterstein S (2002) Probabilistic seismic demand analysis of controlled steel moment-resisting frame structures. Earthquake Engineering \& Structural Dynamics 31:2049-2066. doi: 10.1002/eqe.201

Bazzurro P, Cornell A, Shome N, Carballo J (1998) Three Proposals for Characterizing MDOF Nonlinear Seismic Response. Article in Journal of Structural Engineering. doi: 10.1061/(ASCE)0733-9445(1998)124:11(1281)

Bazzurro P, Cornell CA (1999) Disaggregation of seismic hazard. Bulletin of the Seismological Society of America 89:501-520. doi: $10.1785 / 0120060093$

Boore DM (2003) Simulation of Ground Motion Using the Stochastic Method. In: Seismic Motion, Lithospheric Structures, Earthquake and Volcanic Sources: The Keiiti Aki Volume. Birkhäuser Basel, Basel, pp 635-676

Boore DM, Joyner WB (1997) Site amplifications for generic rock sites. Bulletin of the Seismological Society of America 87:327-341

Bozorgnia Y, Abrahamson NA, Al Atik L, et al (2014) NGA-West2 research project. Earthquake Spectra 30:973-987. doi: 10.1193/072113EQS209M

Bradley B, Dhakal R, MacRae G, Cubrinovski M (2010) Prediction of spatially distributed seismic demands in specific structures:Ground motion and structural response. Earthquake Engineering \& Structural Dynamics 39:501-520. doi: $10.1002 /$ eqe

Bradley BA (2013a) Practice-oriented estimation of the seismic demand hazard using ground motions at few intensity levels. Earthquake Engineering and Structural Dynamics 42:2167-2185. doi: 10.1002/eqe.2319

Bradley BA (2013b) A critical examination of seismic response uncertainty analysis in earthquake engineering. Earthquake Engineering and Structural Dynamics. doi: 10.1002/eqe.2331

Bradley BA, Burks LS, Baker JW (2015) Ground motion selection for simulation-based seismic hazard and structural reliability assessment. Earthquake Engineering \& Structural Dynamics 44:2321-2340. doi: 10.1002/eqe.2588

Bradley BA, Lee DS, Broughton R, Price C (2009) Efficient evaluation of performance-based earthquake engineering equations. Structural Safety 31:65-74. doi: 10.1016/j.strusafe.2008.03.003

Cornell C (2005) On earthquake record selection for nonlinear dynamic analysis. Luis Esteva Symposium

Cornell CA, Jalayer F, Hamburger RO, Foutch DA (2002) Probabilistic Basis for 2000 SAC Federal Emergency Management Agency Steel Moment Frame Guidelines. Journal of Structural Engineering 128:526-533. doi: 10.1061/(ASCE)07339445(2002)128:4(526)

Dall'Asta A, Tubaldi E, Ragni L (2016) Influence of the nonlinear behavior of viscous dampers on the seismic demand hazard of building frames. Earthquake Engineering and Structural Dynamics 45:149-169. doi: 10.1002/eqe.2623

Dávalos H, Miranda E (2019a) Filtered incremental velocity: A novel approach in intensity measures for seismic collapse estimation. Earthquake Engineering and Structural Dynamics. doi: 10.1002/eqe.3205

Dávalos H, Miranda E (2019b) Evaluation of the scaling factor bias influence on the probability of collapse using SA(T1) as the intensity measure. Earthquake Spectra. doi: 10.1193/011018EQS007M

Der Kiureghian A, Fujimura K (2009) Nonlinear stochastic dynamic analysis for performance-based earthquake engineering. Earthquake Engineering and Structural Dynamics. doi: 10.1002/eqe.899

Eads L, Miranda E, Lignos DG (2015) Average spectral acceleration as an intensity measure for collapse risk assessment. Earthquake Engineering and Structural Dynamics. doi: 10.1002/eqe.2575

Eurocode 0 (2002) Eurocode 0 - Basis of structural design. En 1990:2002

FEMA-350 (2000a) Recommended Seismic Design Critria for New Steel Moment-Frame Buildings Fema 350. FEMA-350, Washington, DC. doi: 10.1017/CBO9781107415324.004

FEMA-350 (2000b) FEMA 356: Prestandard and Commentary for the Seimic Rehabilitation of Buildings

FEMA (2005) Improvement of Nonlinear Static Seismic Analysis Procedures. FEMA 440, Federal Emergency Management 
Agency, Washington DC

Franchin P, Cavalieri F, Pinto PE (2012) Validating IM-based methods for probabilistic seismic performance assessment with higher-level non-conditional simulation. In: 15th WCEE Lisboa 2012

Freddi F, Padgett JE, Dall'Asta A (2017) Probabilistic seismic demand modeling of local level response parameters of an RC frame. Bulletin of Earthquake Engineering 15:1-23. doi: 10.1007/s10518-016-9948-x

Gehl P, Douglas J, Seyedi DM (2015) Influence of the number of dynamic analyses on the accuracy of structural response estimates. Earthquake Spectra. doi: 10.1193/102912EQS320M

Gupta A, Krawinkler H (1999) Seismic Demands for Performance Evaluation of Steel Moment Resisting Frame Structures. Doctoral dissertation, Stanford University 1-379

Iervolino I, Spillatura A, Bazzurro P (2018) Seismic reliability of code-conforming Italian buildings. Journal of Earthquake Engineering 1-23. doi: 10.1080/13632469.2018.1540372

Jalayer F, Beck JL (2008) Effects of two alternative representations of ground-motion uncertainty on probabilistic seismic demand assessment of structures. Earthquake Engineering and Structural Dynamics 37:61-79. doi: 10.1002/eqe.745

Jalayer F, Cornell CA (2009) Alternative non-linear demand estimation methods for probability-based seismic assessments. Earthquake Engineering and Structural Dynamics 38:951-972. doi: 10.1002/eqe.876

Jayaram N, Baker JW (2010) Efficient sampling and data reduction techniques for probabilistic seismic lifeline risk assessment. Earthquake Engineering and Structural Dynamics 39:1109-1131

Kazantzi AK, Vamvatsikos D (2015) Intensity measure selection for vulnerability studies of building classes. doi: 10.1002 eqe. 2603

Kramer SL (2003) Geotechnical Earthquake Engineering. Prentice-Hall: Englewood Cliffs, NJ.

Kwong NS, Chopra AK (2016) Evaluation of the exact conditional spectrum and generalized conditional intensity measure methods for ground motion selection. Earthquake Engineering and Structural Dynamics. doi: 10.1002/eqe.2683

Lignos DG, Krawinkler H (2010) Deterioration modeling of steel components in support of collapse prediction of steel moment frames under earthquake loading. Journal of Structural Engineering. doi: 10.1061/(ASCE)ST.1943-541X.0000376

Lin T, Baker JW (2013) Introducing adaptive incremental dynamic analysis: A new tool for linking ground motion selection and structural response assessment. In: Safety, Reliability, Risk and Life-Cycle Performance of Structures and Infrastructures - Proceedings of the 11th International Conference on Structural Safety and Reliability, ICOSSAR 2013

Lin T, Harmsen SC, Baker JW, Luco N (2013) Conditional spectrum computation incorporating multiple causal earthquakes and ground-motion prediction models. Bulletin of the Seismological Society of America. doi: 10.1785/0120110293

Luco N, Cornell CA (2007) Structure-specific scalar intensity measures for near-source and ordinary earthquake ground motions. Earthquake Spectra 23:357-392. doi: 10.1193/1.2723158

Mackie KR, Stojadinović B (2005) Comparison of Incremental Dynamic, Cloud, and Stripe Methods for Computing Probabilistic Seismic Demand Models. In: Structures Congress, ASCE. pp 1-11

Mazzoni S, Mckenna F, Scott MH, Fenves GL (2006) The Open System for Earthquake Engineering Simulation (OpenSEES) User Command-Language Manual

McKenna FT (1997) Object-oriented finite element programming: Frameworks for analysis, algorithms and parallel computing. ProQuest Dissertations and Theses

Ohtori Y, Christenson RE, Spencer BF, Dyke SJ (2004) Benchmark Control Problems for Seismically Excited Nonlinear Buildings. Journal of Engineering Mechanics 130:366-385. doi: 10.1061/(ASCE)0733-9399(2004)130:4(366)

Patelli E (2017) COSSAN: A multidisciplinary software suite for uncertainty quantification and risk management. Handbook of Uncertainty Quantification 1909-1977. doi: 10.1007/978-3-319-12385-1 59

Pinto PE, Giannini R, Franchin P (2004) Seismic reliability analysis of structures. IUSS Press

Porter K (2016) A Beginner's Guide to Fragility, Vulnerability, and Risk. In: University of Colorado Boulder, 92 pp., http://spot.colorado.edu/ porterka/Porter-beginners-guide.pdf

Rezaeian S, Kiureghian A Der (2010) Simulation of synthetic ground motions for specified earthquake and site characteristics. Earthquake Engineering and Structural Dynamics 39:1155-1180. doi: 10.1002/eqe.997

Romão X, Delgado R, Costa A (2011) Assessment of the statistical distributions of structural demand under Earthquake loading. Journal of Earthquake Engineering. doi: 10.1080/13632469.2010.539296

Rubinstein RY, Kroese DP (2017) Simulation and the Monte Carlo Method

Ruiz-García J, Miranda E (2006) Evaluation of residual drift demands in regular multi-storey frames for performance-based seismic assessment. EARTHQUAKE ENGINEERING AND STRUCTURAL DYNAMICS Earthquake Engng Struct Dyn 35:1609-1629. doi: 10.1002/eqe.593

Scozzese F, Dall'Asta A, Tubaldi E (2019) Seismic risk sensitivity of structures equipped with anti-seismic devices with uncertain properties. Structural Safety 77:30-47. doi: 10.1016/J.STRUSAFE.2018.10.003

Scozzese F, Terracciano G, Zona A, Della Corte G, Dall'Asta A, Landolfo R (2018a) Analysis of seismic non-structural damage in single-storey industrial steel buildings. Soil Dynamics and Earthquake Engineering 114:505-519. doi: 10.1016/j.soildyn.2018.07.047

Scozzese F, Terracciano G, Zona A, Della Corte G, Dall'Asta A, Landolfo R (2018b) Modeling and Seismic Response Analysis of Italian Code-Conforming Single-Storey Steel Buildings. Journal of Earthquake Engineering 1-30. doi: 10.1080/13632469.2018.1528913

Seo CY, Karavasilis TL, Ricles JM, Sause R (2014) Seismic performance and probabilistic collapse resistance assessment of steel moment resisting frames with fluid viscous dampers. Earthquake Engineering and Structural Dynamics 43:2135- 
Shapiro SS, Wilk MB (1965) An Analysis of Variance Test for Normality (Complete Samples). Biometrika. doi: 10.2307/2333709

Shome N, Cornell CA (1999) Probabilistic seismic analysis of non-linear structures

Shome N, Cornell CA, Bazzurro P, Carballo JE (1998) Earthquakes, records, and nonlinear responses. Earthquake Spectra 14:469-500. doi: 10.1193/1.1586011

Tubaldi E, Freddi F, Barbato M (2016) Probabilistic seismic demand model for pounding risk assessment. Earthquake Engineering and Structural Dynamics 45:1743-1758. doi: 10.1002/eqe.2725

Tubaldi E, Ragni L, Dall'Asta A (2015) Probabilistic seismic response assessment of linear systems equipped with nonlinear viscous dampers. Earthquake Engineering \& Structural Dynamics 44:101-120. doi: 10.1002/eqe.2461

Vamvatsikos D, Allin Cornell C (2002) Incremental dynamic analysis. Earthquake Engineering and Structural Dynamics 31:491-514. doi: 10.1002/eqe.141

Vetter C, Taflanidis AA (2012) Global sensitivity analysis for stochastic ground motion modeling in seismic-risk assessment. Soil Dynamics and Earthquake Engineering 38:128-143

Yamamoto Y, Baker JW (2013) Stochastic model for earthquake ground motion using wavelet packets. Bulletin of the Seismological Society of America 103:3044-3056. doi: 10.1785/0120120312

Yu YJ, Tsai KC, Li CH, Weng YT, Tsai CY (2013) Earthquake response analyses of a full-scale five-story steel frame equipped with two types of dampers. Earthquake Engineering and Structural Dynamics 42:1301-1320. doi: 10.1002/eqe.2273 